\title{
Nonlinear Dynamics of the 3D Pendulum
}

\author{
Nalin A. Chaturvedi • Taeyoung Lee • \\ Melvin Leok $\cdot$ N. Harris McClamroch
}

Received: 9 July 2008 / Accepted: 23 October 2009 / Published online: 25 September 2010 (C) The Author(s) 2010. This article is published with open access at Springerlink.com

\begin{abstract}
A 3D pendulum consists of a rigid body, supported at a fixed pivot, with three rotational degrees of freedom. The pendulum is acted on by a gravitational force. 3D pendulum dynamics have been much studied in integrable cases that arise when certain physical symmetry assumptions are made. This paper treats the nonintegrable case of the 3D pendulum dynamics when the rigid body is asymmetric and the center of mass is distinct from the pivot location. 3D pendulum full and reduced models are introduced and used to study important features of the nonlinear dynam-
\end{abstract}

Communicated by R. Sepulchre.

N.C., T.L. and N.H.M. have been supported in part by NSF Grant ECS-0244977 and CMS-0555797.

T.L. and M.L. have been supported in part by NSF Grant DMS-0726263, DMS-0747659 and DMS-0747659.

N.A. Chaturvedi $(\bowtie)$

Research and Technology Center, Robert Bosch LLC, Palo Alto, CA 94304, USA

e-mail: nalin.chaturvedi@us.bosch.com

T. Lee

Department of Mechanical and Aerospace Engineering, Florida Institute of Technology, Melbourne, FL, USA

e-mail: taeyoung@fit.edu

M. Leok

Department of Mathematics, University of California at San Diego, La Jolla, CA 92093-0112, USA

e-mail:mleok@math.ucsd.edu

N.H. McClamroch

Department of Aerospace Engineering, The University of Michigan, Ann Arbor, MI 48109-2140,

USA

e-mail:nhm@umich.edu 
ics: conserved quantities, equilibria, relative equilibria, invariant manifolds, local dynamics, and presence of chaotic motions. The paper provides a unified treatment of the $3 \mathrm{D}$ pendulum dynamics that includes prior results and new results expressed in the framework of geometric mechanics. These results demonstrate the rich and complex dynamics of the 3D pendulum.

Keywords Pendulum · Rigid body · Nonlinear dynamics · Attitude · Equilibria • Relative equilibria $\cdot$ Stability $\cdot$ Chaos

Mathematics Subject Classification (2000) $70 \mathrm{E} 17 \cdot 70 \mathrm{~K} 20 \cdot 70 \mathrm{~K} 42 \cdot 65 \mathrm{P} 20$

\section{Introduction}

Pendulum models have been a rich source of examples in nonlinear dynamics and in recent decades, in nonlinear control. The most common rigid pendulum model consists of a mass particle that is attached to one end of a massless, rigid link; the other end of the link is fixed to a pivot point that provides a rotational joint for the link and mass particle. If the link and mass particle are constrained to move within a fixed plane, the system is referred to as a planar 1D pendulum. If the link and mass particle are unconstrained, it is referred to as a spherical 2D pendulum. Planar and spherical pendulum models have been studied, for example, in Astrom and Furuta (2000) and Furuta (2003). Spinning tops, such as the Lagrange top, also constitute another special category of pendulum models (Bruno 2007; Cushman and Bates 1997; Lewis et al. 1992).

Pendulum models are useful for both pedagogical and research reasons. They represent physical mechanisms that can be viewed as simplified academic versions of mechanical systems that arise in, for example, robotics and spacecraft. In addition to their important role in illustrating the fundamental techniques of nonlinear dynamics, pendulum models have motivated new research directions and applications in nonlinear dynamics.

This paper arose out of our continuing research on a laboratory facility, referred to as the Triaxial Attitude Control Testbed (TACT). The TACT was constructed to provide a testbed for physical experiments on attitude dynamics and attitude control. The most important feature of the TACT design is that it is supported by a threedimensional air bearing that serves as an ideal frictionless pivot, allowing nearly unrestricted three degrees of rotation. The TACT has been described in several prior publications (Bernstein et al. 2001; Cho et al. 2001). Issues of nonlinear dynamics for the TACT have been treated in Cho et al. (2001, 2003); issues of nonlinear control for the TACT have been treated in Shen et al. (2004). The present paper is partly motivated by the realization that the TACT is, in fact, a physical implementation of a 3D pendulum.

\section{Description of the 3D Pendulum}

This paper treats 3D pendulum models, some of which were studied by Euler; see Arnold et al. (1988) and Bruno (2007) and references therein for historical back- 
ground. A 3D pendulum is a rigid body supported by a fixed, frictionless pivot, acted on by gravitational forces. The supporting pivot allows three degrees of rotational freedom of the pendulum. Uniform, constant gravity is assumed. The terminology $3 \mathrm{D}$ pendulum refers to the fact that the pendulum is a rigid body with three spatial dimensions and the pendulum has three rotational degrees of freedom.

Two reference frames are introduced. An inertial reference frame has its origin at the pivot; the first two axes lie in the horizontal plane and the third axis is vertical in the direction of gravity. A reference frame fixed to the pendulum body is also introduced. The origin of this body-fixed frame is located at the pivot. In the bodyfixed frame, the inertia tensor of the pendulum is constant. This inertia tensor can be computed from the traditional inertia tensor of a translated frame whose origin is located at the center of mass of the pendulum using the parallel axis theorem. Since the origin of the body-fixed frame is located at the pivot, principal axes with respect to this frame can be defined for which the inertia tensor is diagonal. Note that the center of mass of the 3D pendulum does not necessarily lie on one of the principal axes. Throughout this paper, we assume that the $3 \mathrm{D}$ pendulum is asymmetric, that is its three principal moments of inertia are distinct, and the center of mass of the 3D pendulum is not at the pivot location.

Rotation matrices are used to describe the attitude of the rigid 3D pendulum. A rotation matrix maps a representation of vectors expressed in the body-fixed frame to a representation expressed in the inertial frame. Rotation matrices provide global representations of the attitude of the pendulum, which is why they are utilized here. Other attitude representations, such as exponential coordinates, quaternions, or Euler angles, can also be used following standard descriptions, but each of the representations has the disadvantage of introducing an ambiguity or coordinate singularity. In this paper, the attitude configuration of the pendulum is a rotation matrix $R$ in the special orthogonal group $S O(3)$ defined as

$$
S O(3) \triangleq\left\{R \in \mathbb{R}^{3 \times 3}: R R^{\mathrm{T}}=I_{3 \times 3}, \operatorname{det}(R)=1\right\} .
$$

The associated angular velocity of the pendulum, expressed in the body-fixed frame, is denoted by $\omega$ in $\mathbb{R}^{3}$. The constant inertia tensor of the rigid body pendulum, in the body-fixed frame, is denoted by the symbol $J$. The constant body-fixed vector from the pivot to the center of mass of the pendulum is denoted by $\rho=\left[\begin{array}{lll}\rho_{1} & \rho_{2} & \rho_{3}\end{array}\right]^{\mathrm{T}}$. The symbol $g$ denotes the constant acceleration due to gravity.

Three categories of 3D pendulum models are subsequently introduced and studied. The "full" dynamics of the 3D pendulum is based on Euler's equations that include the gravity moment and the rotational kinematics, expressed in terms of the angular velocity and a rotation matrix; this model describes the dynamics that evolves on $T S O(3)$. Since the gravity moment depends solely on the direction of gravity in the pendulum-fixed frame, it is possible to obtain a reduced model expressed in terms of the angular velocity and a unit vector that defines the direction of gravity in the pendulum-fixed frame; this model describes the dynamics that evolves on $T S O(3) / S^{1}$, and corresponds to the case of Lagrange-Poincaré reduction (Cendra et al. 2001). Since there is a symmetry action given by a rotation about the gravity direction, Lagrange-Routh reduction (Marsden et al. 2000) leads to a reduced model expressed in terms of the unit vector that defines the direction of gravity in 
the pendulum-fixed frame and its derivative; this model describes the dynamics that evolves on $T S^{2}$. Each of these 3D pendulum models provides special insight into the nonlinear dynamics. We develop each of these models in this paper, and we investigate the features of the nonlinear dynamics, namely invariants, equilibria, and stability, for each model.

\section{3D Pendulum Dynamics on TSO(3)}

The dynamics of the $3 \mathrm{D}$ pendulum is given by the Euler equation that includes the moment due to gravity:

$$
J \dot{\omega}=J \omega \times \omega+m g \rho \times R^{\mathrm{T}} e_{3} .
$$

The rotational kinematics equations are

$$
\dot{R}=R \widehat{\omega}
$$

Equations (3.1) and (3.2) define the full dynamics of a rigid pendulum on the tangent bundle $T S O(3)$. Throughout the paper, $e_{1}=(1,0,0)^{\mathrm{T}}, e_{2}=(0,1,0)^{\mathrm{T}}, e_{3}=$ $(0,0,1)^{\mathrm{T}}$. In the inertial frame, $e_{1}$ and $e_{2}$ are assumed to lie in a horizontal plane, while $e_{3}$ is assumed to be in the direction of gravity; consequently $R^{\mathrm{T}} e_{3}$ is the direction of gravity in the pendulum-fixed frame. The cross product notation $a \times b$ for vectors $a$ and $b$ in $\mathbb{R}^{3}$ is

$$
a \times b=\left[a_{2} b_{3}-a_{3} b_{2}, a_{3} b_{1}-a_{1} b_{3}, a_{1} b_{2}-a_{2} b_{1}\right]=\widehat{a} b,
$$

where the skew-symmetric matrix $\widehat{a}$ is defined as

$$
\widehat{a}=\left[\begin{array}{ccc}
0 & -a_{3} & a_{2} \\
a_{3} & 0 & -a_{1} \\
-a_{2} & a_{1} & 0
\end{array}\right] .
$$

\subsection{Integrals of the 3D Pendulum Dynamics}

There are two conserved quantities, or integrals of motion, for the 3D pendulum. First, the total energy, which is the sum of the rotational kinetic energy and the gravitational potential energy, is conserved. In addition, there is a symmetry corresponding to rotations about the gravity direction through the pivot. This symmetry leads to conservation of the component of angular momentum about the gravity direction. These two well-known results, summarized as follows, are easily proved.

\section{Proposition 1 The total energy}

$$
E=\frac{1}{2} \omega^{\mathrm{T}} J \omega-m g \rho^{\mathrm{T}} R^{\mathrm{T}} e_{3},
$$


and the component of the angular momentum vector about the vertical axis through the pivot

$$
h=\omega^{\mathrm{T}} J R^{\mathrm{T}} e_{3} .
$$

are each constant along motions of the $3 D$ pendulum.

Proof This result follows from direct computation of the time derivatives of the total energy and the angular momentum about the vertical axis, by using (3.1) and (3.2).

The assumption that the $3 \mathrm{D}$ pendulum is asymmetric and the center of mass is not located at the pivot implies that there are no nontrivial integrals of motion other than those given in the above proposition. That is, the $3 \mathrm{D}$ pendulum dynamics is not integrable (Cushman and Bates 1997).

We do not further consider the integrable cases of the 3D pendulum dynamics, which includes the free rigid body, the Lagrange top, the Kovalevskaya top, and the Goryachev-Chaplygin top. These integrable cases have been extensively treated in the existing literature (Arnold et al. 1988; Bruno 2007; Cushman and Bates 1997; Karapetyan 2006).

Constant values of the total energy of the 3D pendulum and constant values of the component of angular momentum of the 3D pendulum in the direction of gravity define invariant manifolds of the $3 \mathrm{D}$ pendulum. These invariant manifolds are important characterizations of the 3D pendulum dynamics.

\subsection{Equilibria of the 3D Pendulum}

To understand the dynamics of the 3D pendulum, we study its equilibria. Equating the RHS of (3.1) and (3.2) to zero yields conditions for an equilibrium $\left(R_{\mathrm{e}}, \omega_{\mathrm{e}}\right)$ :

$$
\begin{gathered}
J \omega_{\mathrm{e}} \times \omega_{\mathrm{e}}+m g \rho \times R_{\mathrm{e}}^{\mathrm{T}} e_{3}=0, \\
R_{\mathrm{e}} \widehat{\omega}_{\mathrm{e}}=0 .
\end{gathered}
$$

Since $R_{\mathrm{e}} \in S O(3)$ is non-singular, and $\widehat{r}: \mathbb{R}^{3} \rightarrow \mathbb{R}^{3 \times 3}$ is a linear injection, $R_{\mathrm{e}} \widehat{\omega}_{\mathrm{e}}=0$ if and only if $\omega_{\mathrm{e}}=0$. Substituting $\omega_{\mathrm{e}}=0$ in (3.5), we obtain $\rho \times R_{\mathrm{e}}^{\mathrm{T}} e_{3}=0$. Hence,

$$
R_{\mathrm{e}}^{\mathrm{T}} e_{3}=\frac{\rho}{\|\rho\|}
$$

or

$$
R_{\mathrm{e}}^{\mathrm{T}} e_{3}=-\frac{\rho}{\|\rho\|} .
$$

An attitude $R_{\mathrm{e}}$ is an equilibrium attitude if and only if the direction of gravity resolved in the body-fixed frame, $R_{\mathrm{e}}^{\mathrm{T}} e_{3}$, is collinear with the vector $\rho$. If $R_{\mathrm{e}}^{\mathrm{T}} e_{3}$ is in the same direction as the vector $\rho$, then $\left(R_{\mathrm{e}}, 0\right)$ is a hanging equilibrium of the 3D pendulum; if $R_{\mathrm{e}}^{\mathrm{T}} e_{3}$ is in the opposite direction to the vector $\rho$, then $\left(R_{\mathrm{e}}, 0\right)$ is an inverted equilibrium of the $3 \mathrm{D}$ pendulum. 
Thus, if $R_{\mathrm{e}}$ defines an equilibrium attitude for the 3D pendulum, then a rotation of the $3 \mathrm{D}$ pendulum about the gravity vector by an arbitrary angle is also an equilibrium. Consequently, in $T S O(3)$ there are two disjoint equilibrium manifolds of the $3 \mathrm{D}$ pendulum. The manifold corresponding to the first case in the above description is referred to as the hanging equilibrium manifold, since the center of mass is always below the pivot. The manifold corresponding to the second case in the above description is referred to as the inverted equilibrium manifold, since the center of mass is always above the pivot.

Following (3.7) and (3.8) and the discussion above, we define

$$
\begin{aligned}
& {[R]_{h} \triangleq\left\{R \in S O(3): R^{\mathrm{T}} e_{3}=\frac{\rho}{\|\rho\|}\right\},} \\
& {[R]_{i} \triangleq\left\{R \in S O(3): R^{\mathrm{T}} e_{3}=-\frac{\rho}{\|\rho\|}\right\},}
\end{aligned}
$$

as the hanging attitude manifold and the inverted attitude manifold, respectively.

From (3.7) and (3.8),

$$
\mathrm{H} \triangleq\left\{(R, 0) \in T S O(3): R \in[R]_{h}\right\}
$$

is the manifold of hanging equilibria and

$$
\mathrm{I} \triangleq\left\{(R, 0) \in \operatorname{TSO}(3): R \in[R]_{i}\right\}
$$

is the manifold of inverted equilibria, and these two equilibrium manifolds are clearly disjoint.

\subsection{Local Analysis of the 3D Pendulum near an Equilibrium}

A perturbation from a hanging equilibrium $\left(R_{\mathrm{e}}, 0\right)$ of the $3 \mathrm{D}$ pendulum can be expressed using an exponential representation and a perturbation parameter $\varepsilon$. Let $R^{\varepsilon}(t)$ and $\omega^{\varepsilon}(t)$ represent the perturbed solution, corresponding to initial conditions $R^{\varepsilon}(0)=R_{\mathrm{e}} \exp \varepsilon \widehat{\delta \Theta}$ and $\omega^{\varepsilon}(0)=\varepsilon \delta \omega$, where $\delta \Theta, \delta \omega \in \mathbb{R}^{3}$ are constant vectors. Note that if $\varepsilon=0$, then $\left(R^{0}(0), \omega^{0}(0)\right)=\left(R_{\mathrm{e}}, 0\right)$ and hence

$$
\left(R^{0}(t), \omega^{0}(t)\right) \equiv\left(R_{\mathrm{e}}, 0\right)
$$

for all time $t \in \mathbb{R}$.

The perturbed solution satisfies the perturbed equations of motion for the $3 \mathrm{D}$ pendulum:

$$
\begin{aligned}
J \dot{\omega}^{\varepsilon} & =J \omega^{\varepsilon} \times \omega^{\varepsilon}+m g \rho \times\left(R^{\varepsilon}\right)^{\mathrm{T}} e_{3}, \\
\dot{R}^{\varepsilon} & =R^{\varepsilon} \widehat{\omega^{\varepsilon}} .
\end{aligned}
$$

Next, we differentiate both sides with respect to $\varepsilon$ and substitute $\varepsilon=0$, yielding

$$
J \dot{\omega}_{\varepsilon}^{0}=J \omega_{\varepsilon}^{0} \times \omega^{0}+J \omega^{0} \times \omega_{\varepsilon}^{0}+m g \rho \times\left(R_{\varepsilon}^{0}\right)^{\mathrm{T}} e_{3},
$$




$$
\dot{R}_{\varepsilon}^{0}=R_{\varepsilon}^{0} \widehat{\omega}^{0}+R^{0} \widehat{\omega}_{\varepsilon}^{0}
$$

where the subscripts denote derivatives. Substituting $R^{0}=R_{\mathrm{e}}$ and $\omega^{0}=0$ from (3.13) into (3.16) and (3.17) yields

$$
\begin{aligned}
J \dot{\omega}_{\varepsilon}^{0} & =m g \rho \times\left(R_{\varepsilon}^{0}\right)^{\mathrm{T}} e_{3}, \\
\dot{R}_{\varepsilon}^{0} & =R_{\mathrm{e}} \widehat{\omega}_{\varepsilon}^{0} .
\end{aligned}
$$

Now define perturbation variables $\Delta \omega \triangleq \omega_{\varepsilon}^{0}$ and $\widehat{\Delta \Theta} \triangleq R_{\mathrm{e}}^{\mathrm{T}} R_{\varepsilon}^{0}$. It can be shown that $\Delta \omega=\Delta \dot{\Theta}$. Thus, (3.18) and (3.19) can be written as

$$
J \Delta \ddot{\Theta}-\frac{m g \widehat{\rho}^{2}}{\|\rho\|} \Delta \Theta=0 .
$$

Note that (3.20) can be interpreted as defining a mechanical system with mass matrix $J$, stiffness matrix $-\frac{m g \widehat{\rho}^{2}}{\|\rho\|}$, but no damping. Since $\widehat{\rho}^{2}$ is a negative-semidefinite matrix with two negative eigenvalues and one zero eigenvalue, the stiffness matrix is positive-semidefinite with two positive eigenvalues and one zero eigenvalue. The zero eigenvalue corresponds to rotations about the vertical axis, for which gravity has no influence.

As in Chaturvedi and McClamroch (2007), it can be shown that the linearization given by (3.20) has four imaginary and two zero eigenvalues. Due to the presence of imaginary and zero eigenvalues of the linearized equations, no conclusion can be made about the stability of the hanging equilibrium or the hanging equilibrium manifold of the 3D pendulum. Indeed, the local structure of trajectories in an open neighborhood of the equilibrium is that of a center manifold; there are no stable or unstable manifolds. We next show that the hanging equilibrium manifold of the $3 \mathrm{D}$ pendulum is Lyapunov stable.

Proposition 2 Consider the $3 D$ pendulum model described by (3.1) and (3.2). Then the hanging equilibrium manifold $\mathrm{H}$ given by (3.11) is stable in the sense of Lyapunov.

Proof Consider the following positive-semidefinite function on $\operatorname{TSO}(3)$

$$
V(R, \omega)=\frac{1}{2} \omega^{\mathrm{T}} J \omega+m g\left(\|\rho\|-\rho^{\mathrm{T}} R^{\mathrm{T}} e_{3}\right) .
$$

Note that $V(R, 0)=0$ for all $(R, \omega) \in \mathrm{H}$ and $V(R, \omega)>0$ elsewhere. Furthermore, the derivative along a solution of (3.1) and (3.2) is given by

$$
\begin{aligned}
\dot{V}(R, \omega) & =\omega^{\mathrm{T}} J \dot{\omega}-m g \rho^{\mathrm{T}} \dot{R}^{\mathrm{T}} e_{3} \\
& =\omega^{\mathrm{T}}\left(J \omega \times \omega+m g \rho \times R^{\mathrm{T}} e_{3}\right)-m g \rho^{\mathrm{T}}\left(-\widehat{\omega} R^{\mathrm{T}} e_{3}\right) \\
& =m g\left[\omega^{\mathrm{T}}\left(\rho \times R^{\mathrm{T}} e_{3}\right)+\rho^{\mathrm{T}}\left(\omega \times R^{\mathrm{T}} e_{3}\right)\right]=0 .
\end{aligned}
$$

Thus, $\dot{V}$ is negative-semidefinite on $\operatorname{TSO}(3)$. Also, every sublevel set of the function $V$ is compact. Therefore, the hanging equilibrium manifold $\mathrm{H}$ is Lyapunov stable. 
Similarly, one can linearize the 3D pendulum dynamics about an equilibrium in the inverted equilibrium manifold.

Following the procedure in Chaturvedi and McClamroch (2007), the linearization of the $3 \mathrm{D}$ pendulum about an inverted equilibrium can be shown to result in a linear system that has two positive eigenvalues, two negative eigenvalues and two zero eigenvalues. Thus, the inverted equilibrium has a two-dimensional stable manifold, a two-dimensional unstable manifold and a two-dimensional center manifold. It is clear that due to the presence of the two positive eigenvalues, the inverted equilibrium is unstable. This result is summarized as follows.

Proposition 3 Consider the 3D pendulum model described by (3.1) and (3.2). Then each equilibrium in the inverted equilibrium manifold I given by (3.12) is unstable.

\section{Lagrange-Poincaré Reduced 3D Pendulum Dynamics on $T S O(3) / S^{1}$}

The equations of motion (3.1) and (3.2) for the 3D pendulum are viewed as a model for the dynamics on the tangent bundle TSO(3) (Bloch 2003); these are referred to as the full equations of motion since they characterize the full attitude dynamics of the $3 \mathrm{D}$ pendulum. Since there is a rotational symmetry corresponding to the group of rotations about the vertical axis through the pivot and an associated conserved angular momentum component, it is possible to obtain a lower-dimensional reduced model for the rigid pendulum. This Lagrange-Poincare reduction is based on the fact that the dynamics and kinematics equations can be written in terms of the reduced attitude vector $\Gamma=R^{\mathrm{T}} e_{3} \in S^{2}$, which is the unit vector that expresses the gravity direction in the body-fixed frame (Lewis et al. 1992).

Specifically, let $\Phi_{\theta}$ denote the group action of $S^{1}$ on $S O(3)$, given by $\Phi_{\theta}: S^{1} \times$ $S O(3) \rightarrow S O(3), \Phi_{\theta}(R)=\exp \left(\theta \widehat{e_{3}}\right) R$. This induces an equivalence class by identifying elements of $S O$ (3) that belong to the same orbit; explicitly, for $R_{1}, R_{2} \in S O$ (3), we write $R_{1} \sim R_{2}$ if there exists a $\theta \in S^{1}$ such that $\Phi_{\theta}\left(R_{1}\right)=R_{2}$. The orbit space $S O(3) / S^{1}$ is the set of equivalence classes,

$$
[R] \triangleq\left\{\Phi_{\theta}(R) \in S O(3): \theta \in S^{1}\right\} .
$$

For this equivalence relation, it is easy to see that $R_{1} \sim R_{2}$ if and only if $R_{1}^{\mathrm{T}} e_{3}=R_{2}^{\mathrm{T}} e_{3}$ and hence the equivalence class in (4.1) can alternately, be expressed as

$$
[R] \triangleq\left\{R_{s} \in S O(3): R_{s}^{\mathrm{T}} e_{3}=R^{\mathrm{T}} e_{3}\right\} .
$$

Thus, for each $R \in S O(3),[R]$ can be identified with $\Gamma=R^{\mathrm{T}} e_{3} \in S^{2}$ and hence $S O(3) / S^{1} \cong S^{2}$. This group action induces a projection $\Pi: S O(3) \rightarrow S O(3) / S^{1} \cong S^{2}$ given by $\Pi(R)=R^{T} e_{3}$.

Proposition 4 (Chaturvedi and McClamroch 2007) The dynamics of the $3 D$ pendulum given by (3.1) and (3.2) induces a flow on the quotient space TSO(3)/ $S^{1}$, through the projection $\pi: T S O(3) \rightarrow T S O(3) / S^{1}$ defined as $\pi(R, \Omega)=\left(R^{T} e_{3}, \Omega\right)$, given by the dynamics

$$
J \dot{\omega}=J \omega \times \omega+m g \rho \times \Gamma,
$$


and the kinematics for the reduced attitude

$$
\dot{\Gamma}=\Gamma \times \omega .
$$

Furthermore, $\operatorname{TSO}(3) / S^{1}$ is identified with $S^{2} \times \mathbb{R}^{3}$.

Equations (4.3) and (4.4) are expressed in a non-canonical form; they are referred to as the Lagrange-Poincare reduced attitude dynamics of the $3 \mathrm{D}$ pendulum on $S^{2} \times \mathbb{R}^{3}$.

\subsection{Integrals of the Lagrange-Poincaré Reduced Model}

In a previous section, we presented two integrals of motion for the full model of the $3 \mathrm{D}$ pendulum. In this section we summarize similar well-known results on integrals for the Lagrange-Poincaré reduced model of the 3D pendulum.

Proposition 5 The total energy

$$
E=\frac{1}{2} \omega^{\mathrm{T}} J \omega-m g \rho^{\mathrm{T}} \Gamma,
$$

and the component of the angular momentum vector about the vertical axis through the pivot

$$
h=\omega^{\mathrm{T}} J \Gamma .
$$

are each constant along trajectories of the Lagrange-Poincaré reduced model of the $3 D$ pendulum given by (4.3) and (4.4).

Constant values of the total energy of the 3D pendulum and constant values of the component of angular momentum of the $3 \mathrm{D}$ pendulum in the direction of gravity define invariant manifolds of the Lagrange-Poincaré reduced model of the 3D pendulum. These invariant manifolds are important characterizations of the $3 \mathrm{D}$ pendulum dynamics.

\subsection{Equilibria of the Lagrange-Poincaré Reduced Model}

We study the equilibria of the Lagrange-Poincare reduced equations of motion of the 3D pendulum given by (4.3) and (4.4). Equating the RHS of (4.3) and (4.4) to zero, we obtain conditions that are satisfied by any equilibrium $\left(\Gamma_{\mathrm{e}}, \omega_{\mathrm{e}}\right)$ :

$$
\omega_{\mathrm{e}}=k \Gamma_{\mathrm{e}}, \quad k^{2} J \Gamma_{\mathrm{e}} \times \Gamma_{\mathrm{e}}+m g \rho \times \Gamma_{\mathrm{e}}=0,
$$

for some $k \in \mathbb{R}$. If $k=0$, then $\omega_{\mathrm{e}}=0$ gives two static equilibria of the 3D pendulum; if $k \neq 0$ then relative equilibria (Hernández-Garduño et al. 2005) of the 3D pendulum are obtained.

We assume without loss of generality that the inertia tensor is diagonal, i.e. $J=$ $\operatorname{diag}\left(J_{1}, J_{2}, J_{3}\right)$, where $J_{1}>J_{2}>J_{3}>0$ and $\rho \neq 0$. The following result describes the generic equilibria structure of the Lagrange-Poincaré reduced equations without further assumptions. 
Proposition 6 Consider the Lagrange-Poincaré model of the $3 D$ pendulum given by (4.3) and (4.4). The Lagrange-Poincaré model on $S^{2} \times \mathbb{R}^{3}$ has the following equilibria and relative equilibria:

1. There is a hanging equilibrium: $\left(\frac{\rho}{\|\rho\|}, 0\right) \in S^{2} \times \mathbb{R}^{3}$.

2. There is an inverted equilibrium: $\left(-\frac{\rho}{\|\rho\|}, 0\right) \in S^{2} \times \mathbb{R}^{3}$.

3. There are two relative equilibria in $S^{2} \times \mathbb{R}^{3}$ :

$$
\left(-\frac{J^{-1} \rho}{\left\|J^{-1} \rho\right\|}, \sqrt{\frac{m g}{\left\|J^{-1} \rho\right\|}} J^{-1} \rho\right), \quad\left(-\frac{J^{-1} \rho}{\left\|J^{-1} \rho\right\|},-\sqrt{\frac{m g}{\left\|J^{-1} \rho\right\|}} J^{-1} \rho\right) .
$$

4. There are one-dimensional relative equilibria manifolds in $S^{2} \times \mathbb{R}^{3}$ described by the parameterizations:

$$
\left(-\frac{n_{\alpha}}{\left\|n_{\alpha}\right\|}, \sqrt{\frac{m g}{\left\|n_{\alpha}\right\|}} n_{\alpha}\right), \quad\left(-\frac{n_{\alpha}}{\left\|n_{\alpha}\right\|},-\sqrt{\frac{m g}{\left\|n_{\alpha}\right\|}} n_{\alpha}\right),
$$

where $n_{\alpha}=\left(J-\frac{1}{\alpha} I_{3 \times 3}\right)^{-1} \rho \in \mathbb{R}^{3}$ corresponding to $\alpha \in \mathcal{L}_{i}, i \in\{1,2,3,4,5\}$ with intervals of the reals defined by

$$
\begin{aligned}
& \mathcal{L}_{1}=(-\infty, 0), \quad \mathcal{L}_{2}=\left(0, \frac{1}{J_{1}}\right), \quad \mathcal{L}_{3}=\left(\frac{1}{J_{1}}, \frac{1}{J_{2}}\right), \quad \mathcal{L}_{4}=\left(\frac{1}{J_{2}}, \frac{1}{J_{3}}\right), \\
& \mathcal{L}_{5}=\left(\frac{1}{J_{3}}, \infty\right) .
\end{aligned}
$$

Proof From (4.6), an equilibrium $\left(\Gamma_{\mathrm{e}}, \omega_{\mathrm{e}}\right)$ satisfies

$$
k^{2} J \Gamma_{\mathrm{e}}+m g \rho=k_{1} \Gamma_{\mathrm{e}},
$$

for some constant $k_{1} \in \mathbb{R}$. We solve this equation to obtain the expression for an equilibrium attitude $\Gamma_{\mathrm{e}}$ for two cases; when $k_{1}=0$ and when $k_{1} \neq 0$. The corresponding value of the constant $k$ yields the expression for the equilibrium angular velocity as $\omega_{\mathrm{e}}=k \Gamma_{\mathrm{e}}$.

Equilibria 3: Suppose $k_{1}=0$. It follows that $k \neq 0$ from (4.9). Thus, we have $\Gamma_{\mathrm{e}}=-\frac{m g}{k^{2}} J^{-1} \rho$. Since $\left\|\Gamma_{\mathrm{e}}\right\|=1$, we obtain $k^{2}=m g\left\|J^{-1} \rho\right\|$, which gives (4.7).

Equilibria 1, 2, 4: Suppose $k_{1} \neq 0$. If $k=0$, (4.9) yields the hanging and the inverted equilibrium. Suppose $k \neq 0$. Define $\alpha=\frac{k^{2}}{k_{1}} \in \mathbb{R} \backslash\{0\}$, and $v=k_{1} \Gamma_{\mathrm{e}} \in \mathbb{R}^{3}$. Then (4.9) can be written as

$$
\left(\alpha J-I_{3 \times 3}\right) v=-m g \rho .
$$

Note that for $\alpha \in \mathbb{R} \backslash\left\{0, \frac{1}{J_{1}}, \frac{1}{J_{2}}, \frac{1}{J_{3}}\right\}$ the matrix $\left(J-\frac{1}{\alpha} I_{3 \times 3}\right)$ is invertible. Then (4.10) can be solved to obtain $v=-\frac{m g}{\alpha}\left(J-\frac{1}{\alpha} I_{3 \times 3}\right)^{-1} \rho$. Since $\left\|\Gamma_{\mathrm{e}}\right\|=1$, we have $\|v\|=$ $\left\|k_{1} \Gamma_{\mathrm{e}}\right\|=\left|k_{1}\right|$. We consider two sub-cases; when $k_{1}>0$, and $k_{1}<0$. 
If $k_{1}>0$, we have $k_{1}=\|v\|$ and $\alpha>0$. Thus, we obtain the expression for values of the equilibria attitudes as

$$
\Gamma_{\mathrm{e}}=\frac{v}{\|v\|}=\frac{-\frac{1}{\alpha} n_{\alpha}}{\left|-\frac{1}{\alpha}\right|\left\|n_{\alpha}\right\|}=-\frac{n_{\alpha}}{\left\|n_{\alpha}\right\|},
$$

where $n_{\alpha}=\left(J-\frac{1}{\alpha} I_{3 \times 3}\right)^{-1} \rho \in \mathbb{R}^{3}$. Since $k^{2}=\alpha k_{1}=\alpha\|v\|$, we obtain the expression for values of the equilibria angular velocities as

$$
\omega_{\mathrm{e}}=k \Gamma_{\mathrm{e}}=\mp \sqrt{\alpha\|v\|} \frac{n_{\alpha}}{\left\|n_{\alpha}\right\|}=\mp \sqrt{\frac{m g}{\left\|n_{\alpha}\right\|}} n_{\alpha} .
$$

Thus, (4.11) and (4.12) correspond to the families of equilibria given by (4.8) for $\alpha>0$.

Similarly, if $k_{1}<0$, we have $k_{1}=-\|v\|, \alpha<0$, and $k^{2}=-\alpha\|v\|$. Thus, we obtain the expression for the values of the relative equilibria as

$$
\Gamma_{\mathrm{e}}=-\frac{v}{\|v\|}=\frac{\frac{1}{\alpha} n_{\alpha}}{\left|-\frac{1}{\alpha}\right|\left\|n_{\alpha}\right\|}=-\frac{n_{\alpha}}{\left\|n_{\alpha}\right\|}, \quad \omega_{\mathrm{e}}=\mp \sqrt{\frac{m g}{\left\|n_{\alpha}\right\|}} n_{\alpha},
$$

which corresponds to the families of equilibria given by (4.8) for $\alpha<0$.

Equation (4.10) for $\alpha \in \mathbb{R}$ characterizes the equilibria of (4.3) and (4.4). Condition (4.8) presents solutions of (4.10) for all $\alpha \in \mathbb{R} \backslash\left\{0, \frac{1}{J_{1}}, \frac{1}{J_{2}}, \frac{1}{J_{3}}\right\}$. The parameter value $\alpha=0$ yields the hanging and the inverted equilibria.

The first three statements in Proposition 6 are self-explanatory. The fourth statement describes parameterizations for one-dimensional relative equilibria manifolds; these parameterizations are expressed in terms of the real parameter $\alpha$ that lies in one of the four defined intervals. The intervals exclude only the real values $0, \frac{1}{J_{1}}, \frac{1}{J_{2}}, \frac{1}{J_{3}}$ at which the parameterizations are not continuous.

Denote

$$
\Gamma_{h} \triangleq \frac{\rho}{\|\rho\|}, \quad \Gamma_{i} \triangleq-\frac{\rho}{\|\rho\|}, \quad \text { and } \quad \Gamma_{\infty} \triangleq-\frac{J^{-1} \rho}{\left\|J^{-1} \rho\right\|} .
$$

Then it follows from Proposition 6 that $\left(\Gamma_{h}, 0\right)$ and $\left(\Gamma_{i}, 0\right)$ are equilibria of the Lagrange-Poincaré reduced model of the 3D pendulum. These are called the hanging equilibrium and the inverted equilibrium of the Lagrange-Poincaré reduced model, respectively. We refer to $\Gamma_{h}$ and to $\Gamma_{i}$ as the hanging equilibrium attitude and the inverted equilibrium attitude, respectively. As shown subsequently, $\Gamma_{\infty}$ is a vector that is used to define the limit of two of the relative equilibrium manifolds in (4.8).

Convergence properties of the relative equilibria as the parameter $\alpha$ tends to each of the distinguished real values $0, \frac{1}{J_{1}}, \frac{1}{J_{2}}, \frac{1}{J_{3}}$ are addressed in the following proposition.

Proposition 7 The equilibria and relative equilibria of the $3 D$ pendulum given in the parameterizations (4.8) have the following convergence properties: 
1. $\lim _{\alpha \rightarrow \infty} \Gamma_{e}=\Gamma_{\infty}, \quad \lim _{\alpha \rightarrow \infty} \omega_{e}=\mp \sqrt{\frac{m g}{\left\|J^{-1} \rho\right\|}} J^{-1} \rho$;

2. $\lim _{\alpha \rightarrow 0^{-}} \Gamma_{e}=\Gamma_{i}, \quad \lim _{\alpha \rightarrow 0^{-}} \omega_{e}=0$;

3. $\lim _{\alpha \rightarrow 0^{+}} \Gamma_{e}=\Gamma_{h}, \quad \lim _{\alpha \rightarrow 0^{+}} \omega_{e}=0$;

4. for $i \in\{1,2,3\}: \lim \Gamma_{e}=\operatorname{sgn}\left(\rho_{i}\right) e_{i}$, $\alpha \rightarrow{\frac{1}{J_{i}}}^{-}$

5. for $i \in\{1,2,3\}: \lim _{\alpha \rightarrow \frac{1}{J}_{i}^{+}} \Gamma_{e}=-\operatorname{sgn}\left(\rho_{i}\right) e_{i}, \quad \lim _{\alpha \rightarrow{\frac{1}{J_{i}}}^{+}} \omega_{e}= \pm \infty e_{i}$.

Proof Consider the limiting case when $\alpha \rightarrow \infty$. Since $\alpha>0$, we have, from (4.11) and (4.12),

$$
\lim _{\alpha \rightarrow \infty} \Gamma_{\mathrm{e}}=\lim _{\alpha \rightarrow \infty}-\frac{n_{\alpha}}{\left\|n_{\alpha}\right\|}=\lim _{\alpha \rightarrow \infty}-\frac{\left(J-I_{3 \times 3} / \alpha\right)^{-1} \rho}{\left\|\left(J-I_{3 \times 3} / \alpha\right)^{-1} \rho\right\|}=-\frac{J^{-1} \rho}{\left\|J^{-1} \rho\right\|}=\Gamma_{\infty} .
$$

Similarly,

$$
\lim _{\alpha \rightarrow \infty} \omega_{\mathrm{e}}=\lim _{\alpha \rightarrow \infty} \sqrt{\frac{m g}{\left\|n_{\alpha}\right\|}} n_{\alpha}=\sqrt{\frac{m g}{\left\|J^{-1} \rho\right\|}} J^{-1} \rho
$$

Thus, as $\alpha \rightarrow \infty$, the relative equilibria converge to the relative equilibria given in (4.7). It can be similarly shown that the relative equilibria also converge to the relative equilibria given in (4.7) when $\alpha \rightarrow-\infty$.

Next, consider (4.8) as $\alpha \rightarrow 0$. Expressing $n_{\alpha}=\alpha\left(\alpha J-I_{3 \times 3}\right)^{-1} \rho$, we obtain

$$
\lim _{\alpha \rightarrow 0^{-}} \Gamma_{\mathrm{e}}=\lim _{\alpha \rightarrow 0^{-}}-\frac{n_{\alpha}}{\left\|n_{\alpha}\right\|}=\lim _{\alpha \rightarrow 0^{-}}-\frac{\alpha\left(\alpha J-I_{3 \times 3}\right)^{-1} \rho}{\left\|\alpha\left(\alpha J-I_{3 \times 3}\right)^{-1} \rho\right\|}=-\frac{\rho}{\|\rho\|}=\Gamma_{\mathrm{i}},
$$

which corresponds to the inverted attitude. Similarly,

$$
\lim _{\alpha \rightarrow 0^{+}} \Gamma_{\mathrm{e}}=\lim _{\alpha \rightarrow 0^{+}}-\frac{n_{\alpha}}{\left\|n_{\alpha}\right\|}=\lim _{\alpha \rightarrow 0^{+}}-\frac{\alpha\left(\alpha J-I_{3 \times 3}\right)^{-1} \rho}{\left\|\alpha\left(\alpha J-I_{3 \times 3}\right)^{-1} \rho\right\|}=\frac{\rho}{\|\rho\|}=\Gamma_{\mathrm{h}},
$$

which corresponds to the hanging attitude. Also,

$$
\lim _{\alpha \rightarrow 0^{-}} \omega_{\mathrm{e}}=\lim _{\alpha \rightarrow 0^{-}} \sqrt{\frac{m g}{\left\|n_{\alpha}\right\|}} n_{\alpha}=\lim _{\alpha \rightarrow 0^{-}} \sqrt{m g\left\|n_{\alpha}\right\|} \frac{n_{\alpha}}{\left\|n_{\alpha}\right\|}=-\frac{\rho}{\|\rho\|} \lim _{\alpha \rightarrow 0^{-}} \sqrt{m g\|\alpha \rho\|}=0 .
$$

Similarly,

$$
\lim _{\alpha \rightarrow 0^{+}} \omega_{\mathrm{e}}=0 .
$$

Now, it follows that

$$
\lim _{\alpha \rightarrow 1 / J_{1}-} \Gamma_{e}=\lim _{\alpha \rightarrow 1 / J_{1}-}-\frac{\left[\frac{\rho_{1}}{J_{1}-1 / \alpha}, \frac{\rho_{2}}{J_{2}-J_{1}}, \frac{\rho_{3}}{J_{3}-J_{1}}\right]^{T}}{\left\|\left[\frac{\rho_{1}}{J_{1}-1 / \alpha}, \frac{\rho_{2}}{J_{2}-J_{1}}, \frac{\rho_{3}}{J_{3}-J_{1}}\right]\right\|}=\operatorname{sgn}\left(\rho_{1}\right) e_{1},
$$


and

$\lim _{\alpha \rightarrow 1 / J_{1}-} \omega_{\mathrm{e}}=\lim _{\alpha \rightarrow 1 / J_{1}-} \sqrt{m g} \frac{\left[\frac{\rho_{1}}{J_{1}-1 / \alpha}, \frac{\rho_{2}}{J_{2}-J_{1}}, \frac{\rho_{3}}{J_{3}-J_{1}}\right]^{T}}{\sqrt{\left\|\left[\frac{\rho_{1}}{J_{1}-1 / \alpha}, \frac{\rho_{2}}{J_{2}-J_{1}}, \frac{\rho_{3}}{J_{3}-J_{1}}\right]^{T}\right\|}}=\left[-\operatorname{sgn}\left(\rho_{1}\right) \infty, 0,0\right]^{T}$.

The remaining cases are analyzed in a similar way.

If additional assumptions are made about the location of the center of mass, then additional relative equilibria exist. The following results summarize this situation.

Proposition 8 Consider the 3D pendulum and assume that the location of the center of mass vector $\rho$ satisfies the indicated property.

1. Assume there is exactly one index $i \in\{1,2,3\}$ for which $\rho_{i}=0$. There are onedimensional relative equilibria manifolds in $S^{2} \times \mathbb{R}^{3}$ described by the parameterizations:

$$
\left(-\frac{p_{i}}{\left\|p_{i}\right\|}, \sqrt{\frac{m g}{\left\|p_{i}\right\|}} p_{i}\right), \quad\left(-\frac{p_{i}}{\left\|p_{i}\right\|},-\sqrt{\frac{m g}{\left\|p_{i}\right\|}} p_{i}\right) \quad \text { for any } \gamma \in \mathbb{R},
$$

where $p_{1}=\left(\gamma, \frac{\rho_{2}}{J_{2}-J_{1}}, \frac{\rho_{3}}{J_{3}-J_{1}}\right), p_{2}=\left(\frac{\rho_{1}}{J_{1}-J_{2}}, \gamma, \frac{\rho_{3}}{J_{3}-J_{2}}\right), p_{3}=\left(\frac{\rho_{1}}{J_{1}-J_{3}}, \frac{\rho_{2}}{J_{2}-J_{3}}, \gamma\right)$.

2. Assume $\rho_{i}=0$ for exactly two indices $i \in\{1,2,3\}$. There are one-dimensional relative equilibria manifolds in $S^{2} \times \mathbb{R}^{3}$ described by the parameterizations:

$$
\left(e_{i}, \gamma e_{i}\right),\left(-e_{i}, \gamma e_{i}\right), \quad \text { for any } \gamma \in \mathbb{R} \text {. }
$$

Proof Consider the first statement. It is easy to see that for $\alpha=1 / J_{1},(4.10)$ has a solution iff $\rho_{1}=0$. In this case, (4.10) can be written as

$$
\left[\begin{array}{ccc}
0 & 0 & 0 \\
0 & J_{2}-J_{1} & 0 \\
0 & 0 & J_{3}-J_{1}
\end{array}\right] v=-m g J_{1}\left[\begin{array}{c}
0 \\
\rho_{2} \\
\rho_{3}
\end{array}\right]
$$

Since $\alpha>0$, it can be shown as in (4.11) that $\Gamma_{\mathrm{e}}=-\frac{p_{1}}{\left\|p_{1}\right\|}$ and $\omega_{\mathrm{e}}= \pm \sqrt{\frac{m g}{\left\|p_{1}\right\|}} p_{1}$, where $p_{1}=\left(\gamma, \frac{\rho_{2}}{J_{2}-J_{1}}, \frac{\rho_{3}}{J_{3}-J_{1}}\right)$ and $\gamma \in \mathbb{R}$. Similarly, one can obtain solutions of (4.10) for the case where $\alpha=1 / J_{2}$ and $\alpha=1 / J_{3}$ iff $\rho_{2}=0$ and $\rho_{3}=0$, respectively.

Now consider the second statement. The assumption guarantees that the vector $\rho$ can be expressed as $\rho=s e_{i}$, for some $s \in \mathbb{R}$. Then for all $\alpha \in \mathbb{R} \backslash\left\{0, \frac{1}{J_{1}}, \frac{1}{J_{2}}, \frac{1}{J_{3}}\right\}$ it follows that $n_{\alpha}=\frac{1}{\alpha}\left(\alpha J-I_{3 \times 3}\right)^{-1} \rho=\frac{s}{\alpha\left(\alpha J_{i}-1\right)} e_{i}$. Thus the description of the relative equilibria given in (4.8) can be parameterized as $\left\{\left(e_{i}, \gamma e_{i}\right),\left(-e_{i}, \gamma e_{i}\right)\right\}$ for $\gamma \in \mathbb{R}$.

This proposition includes the result for an asymmetric 3D pendulum that if the center of mass lies on a principal axis, then there exist relative equilibria defined by a constant angular velocity vector with arbitrary magnitude and direction that is aligned with that principal axis. 
The geometric description of relative equilibria of the Lagrange-Poincaré model given in Proposition 6 and Proposition 8 provides important insight into the equilibria structure of the 3D pendulum. This geometric description is consistent with the implicit characterization of relative equilibria given in Maddocks (1991).

We now relate the equilibria structure for the Lagrange-Poincaré reduced model, as described in this section, to the equilibria structure for the full model, as described in the previous section. Let $\left(R_{\mathrm{e}}, 0\right)$ denote an equilibrium in either the hanging equilibrium manifold or the inverted equilibrium manifold of the full equations (3.1) and (3.2) and $\pi: T S O(3) \rightarrow T S O(3) / S^{1}$ be the projection as in Proposition 4 . Then it can be shown that either $\pi\left(R_{\mathrm{e}}, 0\right)=\left(\Gamma_{h}, 0\right)$ or $\pi\left(R_{\mathrm{e}}, 0\right)=\left(\Gamma_{i}, 0\right)$. Thus, we obtain the following.

Proposition 9 (Chaturvedi and McClamroch 2007) The hanging equilibrium manifold and the inverted equilibrium manifold of the $3 D$ pendulum given by the full equations (3.1) and (3.2) are identified with the hanging equilibrium $\left(\Gamma_{h}, 0\right)$ and the inverted equilibrium $\left(\Gamma_{i}, 0\right)$ of the Lagrange-Poincaré reduced equations given by (4.3) and (4.4).

\subsection{Visualization of Equilibria and Relative Equilibria}

We examine the equilibrium structure of a particular 3D pendulum model, demonstrating how this equilibrium structure can be visualized. We choose an elliptic cylinder with its semi-major axis $a=0.8 \mathrm{~m}$, semi-minor axis $b=0.2 \mathrm{~m}$, and height $0.6 \mathrm{~m}$. The pivot point is located at the surface of the upper ellipse, and it is offset from the center by $\left[-\frac{a}{6}, \frac{b}{2}, 0\right]$. The inertia tensor is given by $J=$ $\operatorname{diag}(0.3061,0.2136,0.1159) \mathrm{kg} \cdot \mathrm{m}^{2}$ and the vector from the pivot to the mass center is $\rho=[-0.0160,0.2077,0.2727] \mathrm{m}$.

Figures 1(a)-1(c) show the equilibrium and relative equilibrium attitudes on $S^{2}$. Figure 1(a) provides a 3D perspective; Fig. 1(b) provides a top view, with the inverted equilibrium attitude located at the center and Fig. 1(c) provides a bottom view, with the hanging equilibrium attitude located at the center.

The inverted equilibrium attitude $\Gamma_{i}$ and the limiting relative equilibrium attitude $\Gamma_{\infty}$ described by (4.7) are denoted by circles in Fig. 1(b); the hanging equilibrium attitude $\Gamma_{h}$ is denoted by a circle in Fig. 1(c). The one-dimensional manifolds of relative equilibrium attitudes described in (4.8) are shown by five curve segments illustrated by the solid lines corresponding to $\alpha \in \mathcal{L}_{i}$ for $i \in\{1,2,3,4,5\}$, where the increasing value of $\alpha$ is denoted by arrows on each segment.

The relative equilibrium attitudes for $\alpha \in \mathcal{L}_{1}=(-\infty, 0)$ are shown by a segment of a thick line in Fig. 1(b), which starts from $\Gamma_{\infty}$, and converges to the inverted equilibrium attitude $\Gamma_{i}$ as $\alpha$ increases, according to Proposition 7. For $\alpha \in \mathcal{L}_{2}=$ $\left(0,1 / J_{1}\right)$, the line of relative equilibrium attitudes starts from $\Gamma_{h}$, and ends at $-e_{1}$ in Fig. 1(c), since $\rho_{1}<0$ for the given pendulum model. Similarly, for $\alpha \in \mathcal{L}_{3}=$ $\left(1 / J_{1}, 1 / J_{2}\right)$, the line of relative equilibrium attitudes begins at $e_{1}$ and tends to $e_{2}$, and for $\alpha \in \mathcal{L}_{4}=\left(1 / J_{2}, 1 / J_{3}\right)$ the line of relative equilibrium attitudes begins from $-e_{2}$ and ends at $e_{3}$, as $\alpha$ increases. The relative equilibrium attitudes for $\alpha \in \mathcal{L}_{5}=$ $\left(1 / J_{3}, \infty\right)$ are shown by a segment of a thin line in Fig. 1(b), which begins at $-e_{3}$, and 

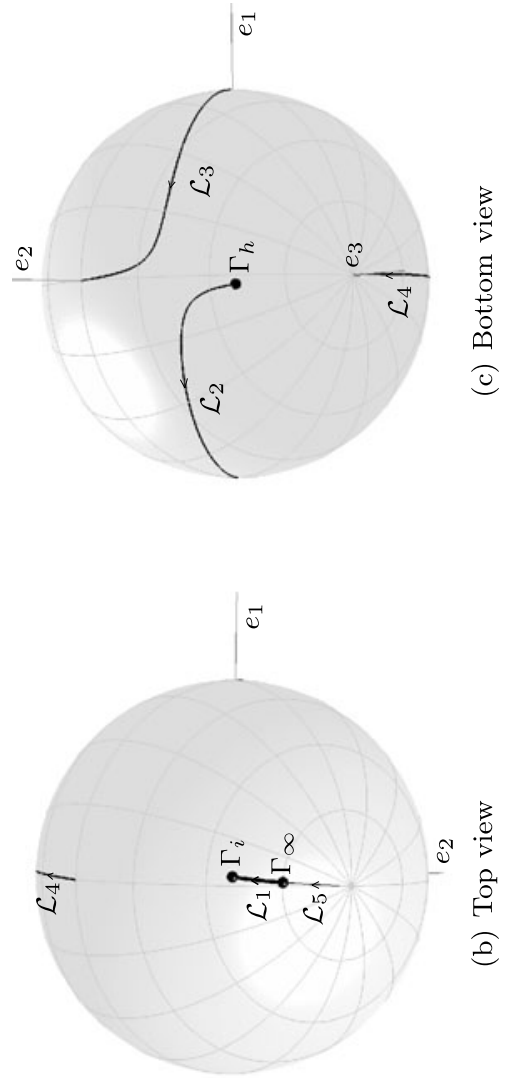

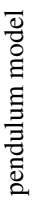

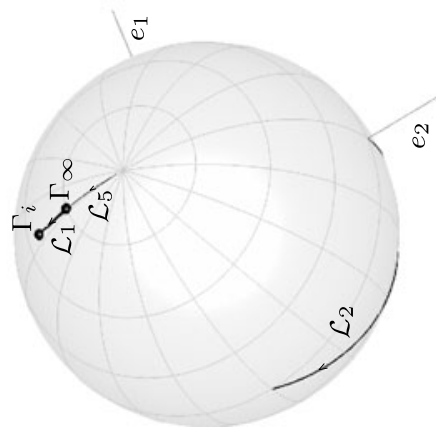

3
0
5
0
0
0
0
0
0

ले

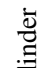

:

(3)

穿

강

高

家 
ends at $\Gamma_{\infty}$. Therefore, the line of relative equilibrium attitudes for $\alpha \in \mathcal{L}_{1}$ and the line of relative equilibrium attitudes for $\alpha \in \mathcal{L}_{5}$ are connected. Since no component of the center of mass vector vanishes, there are no additional relative equilibria.

In summary, we have provided a graphical illustration of the hanging equilibrium attitude, the inverted equilibrium attitude, the relative equilibrium attitude given by (4.7), and the four mutually disjoint one-dimensional relative equilibrium attitude curve segments.

\subsection{Local Analysis of the Lagrange-Poincaré Reduced Model Near an Equilibrium}

In the last section, we showed that the Lagrange-Poincare reduced model of the 3D pendulum has exactly two static equilibria, namely the hanging equilibrium and the inverted equilibrium. As stated in Proposition 9, these equilibria correspond to the disjoint equilibrium manifolds of the full equations of the 3D pendulum.

We focus on these static equilibria of the Lagrange-Poincaré reduced equations. The identification mentioned in Proposition 9 relates properties of the equilibrium manifolds of the full equations and the equilibria of the Lagrange-Poincaré reduced equations. We can deduce the stability of the hanging and the inverted equilibrium manifolds of the full equations from the stability property of the hanging equilibrium and the inverted equilibrium of the Lagrange-Poincaré reduced equations.

Proposition 10 The hanging equilibrium $\left(\Gamma_{h}, 0\right)=\left(\frac{\rho}{\|\rho\|}, 0\right)$, of the LagrangePoincaré reduced dynamics of the $3 D$ pendulum described by (4.3) and (4.4) is stable in the sense of Lyapunov.

Proof Consider the Lyapunov function

$$
V(\Gamma, \omega)=\frac{1}{2} \omega^{\mathrm{T}} J \omega+m g\left(\|\rho\|-\rho^{\mathrm{T}} \Gamma\right) .
$$

Note that $V\left(\Gamma_{h}, 0\right)=0$ and $V(\Gamma, \omega)>0$ elsewhere. Furthermore, the derivative along a solution of (4.3) and (4.4) is given by

$$
\begin{aligned}
\dot{V}(\Gamma, \omega) & =\omega^{\mathrm{T}} J \dot{\omega}-m g \rho^{\mathrm{T}} \dot{\Gamma} \\
& =\omega^{\mathrm{T}}(J \omega \times \omega+m g \rho \times \Gamma)-m g \rho^{\mathrm{T}}(\Gamma \times \omega) \\
& =\omega^{\mathrm{T}} m g \rho \times \Gamma-m g \rho^{\mathrm{T}} \Gamma \times \omega=0 .
\end{aligned}
$$

Thus, the hanging equilibrium is Lyapunov stable.

Note that combining Proposition 10 with Proposition 9 immediately confirms the stability result for the hanging equilibrium manifold in Proposition 2.

We next examine the local properties of the Lagrange-Poincaré reduced equations of the 3D pendulum near the inverted equilibrium $\left(\Gamma_{i}, 0\right)$. Consider the linearization of (4.3)-(4.4) about an equilibrium $\left(\Gamma_{i}, 0\right)=\left(R_{\mathrm{e}}^{\mathrm{T}} e_{3}, 0\right)$, where $\left(R_{\mathrm{e}}, 0\right)$ is an equilibrium in the inverted equilibrium manifold $I$. 
As in Chaturvedi and McClamroch (2007), the inverted equilibrium of the Lagrange-Poincaré reduced equations can be shown to have two negative eigenvalues, two positive eigenvalues and a zero eigenvalue. Thus, the inverted equilibrium $\left(\Gamma_{i}, 0\right)$ is unstable and locally there exists a two-dimensional stable manifold, a twodimensional unstable manifold and a one-dimensional center manifold. This is summarized as follows.

Proposition 11 The inverted equilibrium $\left(\Gamma_{i}, 0\right)=\left(-\frac{\rho}{\|\rho\|}, 0\right)$ of the LagrangePoincaré reduced model of the 3D pendulum described by (4.3) and (4.4) is unstable.

Note that combining Proposition 11 with Proposition 9 immediately recovers the result that the inverted equilibrium manifold I of the full equations for the $3 \mathrm{D}$ pendulum given by (3.1)-(3.2) is unstable.

We have analyzed the local stability properties of the hanging equilibrium and of the inverted equilibrium of the Lagrange-Poincaré model. We have not analyzed local stability properties of other equilibrium solutions, namely the relative equilibria of the Lagrange-Poincaré model. Such an analysis can easily be carried out using the constrained variations that respect the Lie group structure of the attitude configurations following the methods introduced in this paper. Alternatively, an analysis of the stability of the relative equilibria of the Lagrange-Poincaré model has been provided in Maddocks (1991) using constrained second variation methods that enforce the Lie group constraints using Lagrange multipliers.

\section{Lagrange-Routh Reduced 3D Pendulum Dynamics on $T S^{2}$}

In the previous sections we studied the full dynamics and the Lagrange-Poincare reduced dynamics of the $3 \mathrm{D}$ pendulum. These involved the study of the dynamics of the 3D pendulum on $T S O(3)$ and on $S^{2} \times \mathbb{R}^{3}$, respectively, using variables $(R, \omega)$ and $(\Gamma, \omega)$ to express the equations of motion. In this section, we present Lagrange-Routh reduction of the $3 \mathrm{D}$ pendulum, and we study the equations of motion that describe the evolution of $(\Gamma, \dot{\Gamma}) \in T S^{2}$.

\subsection{Lagrange-Routh Reduction of the 3D Pendulum}

Lagrange-Routh reduction involves identifying trajectories that are related by the symmetry group action, and further restricting the dynamics to a level set of the associated momentum map. Since the symmetry group is abelian, the dynamics on the configuration manifold $S O(3)$ can be reduced to the shape manifold, which is the quotient manifold associated with the symmetry action. The resulting equations of motion on the quotient manifold are described not in terms of the Lagrangian but in terms of the Routhian (Holmes and Marsden 1983; Lewis et al. 1992; Marsden et al. 2000).

The $3 \mathrm{D}$ pendulum has a $S^{1}$ symmetry given by a rotation about the vertical axis. The symmetry action $\Phi_{\theta}: S^{1} \times S O(3) \rightarrow S O(3)$ is given by

$$
\Phi_{\theta}(R)=\exp \left(\theta \widehat{e}_{3}\right) R
$$


for $\theta \in S^{1}$ and $R \in S O(3)$. It can be shown that the Lagrangian of the 3D pendulum is invariant under this symmetry action. Thus, the configuration manifold $S O(3)$ is reduced to the shape manifold $S O(3) / S^{1} \cong S^{2}$, and the reduced dynamics of the $3 \mathrm{D}$ pendulum is described on the tangent bundle $T S^{2}$. This reduction procedure is interesting and challenging, since the projection $\Pi: S O(3) \rightarrow S^{2}$ given by $\Pi(R)=$ $R^{T} e_{3}$ together with the symmetry action has a nontrivial principal bundle structure. In other words, the angle of the rotation about the vertical axis is not a global cyclic variable.

Here we present expressions for the Routhian and the reduced equations of motion. The detailed description and development can be found in Appendix.

Proposition 12 (Marsden et al. 2000) We identify the Lie algebra of $S^{1}$ with $\mathbb{R}$. For $(R, \omega) \in T_{R} S O(3)$, the momentum map $\mathbf{J}: T S O(3) \rightarrow \mathbb{R}^{*}$, the locked inertia tensor $\mathbb{I}(R): \mathbb{R} \rightarrow \mathbb{R}^{*}$, and the mechanical connection $\mathcal{A}: T S O(3) \rightarrow \mathbb{R}$ for the $3 D$ pendulum are given as follows:

$$
\begin{aligned}
\mathbf{J}(R, \hat{\omega}) & =e_{3}^{T} R J \omega, \\
\mathbb{I}(R) & =e_{3}^{T} R J R^{T} e_{3}, \\
\mathcal{A}(R, \hat{\omega}) & =\frac{e_{3}^{T} R J \omega}{e_{3}^{T} R J R^{T} e_{3}} .
\end{aligned}
$$

The value of the momentum map $\mu=\mathbf{J}(R, \hat{\omega})$ corresponds to the vertical component of the angular momentum. Noether's theorem states that the symmetry of the Lagrangian implies conservation of the corresponding momentum map. This is an alternative method of establishing the invariance properties of the $3 \mathrm{D}$ pendulum dynamics, as opposed to the direct computation in Sect. 3.1.

Based on the above expressions, Lagrange-Routh reduction is carried out to obtain the following result.

Proposition 13 For a given value of the momentum map $\mu$, the Routhian of the $3 D$ pendulum is given by

$$
R^{\mu}(\Gamma, \dot{\Gamma})=\frac{1}{2}(\dot{\Gamma} \times \Gamma) \cdot J(\dot{\Gamma} \times \Gamma)-\frac{1}{2}\left(b^{2}+v^{2}\right)(\Gamma \cdot J \Gamma)+m g \Gamma \cdot \rho,
$$

where $b=\frac{J \Gamma \cdot(\dot{\Gamma} \times \Gamma)}{\Gamma \cdot J \Gamma}, v=\frac{\mu}{\Gamma \cdot J \Gamma}$, and the magnetic two form can be written as

$$
\beta_{\mu}(\Gamma \times \eta, \Gamma \times \zeta)=-\frac{\mu}{(\Gamma \cdot J \Gamma)^{2}}\left[-(\Gamma \cdot J \Gamma) \operatorname{tr}[J]+2\|J \Gamma\|^{2}\right] \Gamma \cdot(\eta \times \zeta) .
$$

The Routhian satisfies the Euler-Lagrange equation, with the magnetic term given by

$$
\delta \int_{0}^{T} R^{\mu}(\Gamma, \dot{\Gamma}) \mathrm{d} t=\int_{0}^{T} \mathbf{i}_{\dot{\Gamma}} \beta_{\mu}(\delta \Gamma) \mathrm{d} t .
$$

This yields the reduced equation of motion on $T S^{2}$ :

$$
\ddot{\Gamma}=-\|\dot{\Gamma}\|^{2} \Gamma+\Gamma \times \Sigma(\Gamma, \dot{\Gamma}),
$$


where

$$
\begin{aligned}
\Sigma(\Gamma, \dot{\Gamma})= & b \dot{\Gamma}+J^{-1}[(J(\dot{\Gamma} \times \Gamma)-b J \Gamma) \times((\dot{\Gamma} \times \Gamma)-b \Gamma) \\
& \left.+v^{2} J \Gamma \times \Gamma-m g \Gamma \times \rho-c \dot{\Gamma}\right], \\
c= & v\left\{\operatorname{tr}[J]-2 \frac{\|J \Gamma\|^{2}}{\Gamma \cdot J \Gamma}\right\}, \quad b=\frac{J \Gamma \cdot(\dot{\Gamma} \times \Gamma)}{\Gamma \cdot J \Gamma}, \quad v=\frac{\mu}{\Gamma \cdot J \Gamma} .
\end{aligned}
$$

Proof See Appendix.

The function $\Sigma(\Gamma, \dot{\Gamma})$ is an exceedingly complicated function of its arguments. This makes direct analysis of (5.7) a challenge.

\subsection{Lagrange-Routh Reconstruction of the 3D Pendulum}

For a given value of the momentum map $\mu$, let $\Gamma(t) \in S^{2}$ be a curve in the reduced space $S^{2}$ satisfying the Lagrange-Routh reduced model given by (5.7). The reconstruction procedure is to find the curve $R(t) \in S O(3)$ that satisfies $\Pi(R(t))=\Gamma(t)$ and $\mathbf{J}\left(R(t), R(t)^{T} \dot{R}(t)\right)=\mu$.

This can be achieved in two steps. First, we choose any curve $\tilde{R}(t) \in S O(3)$ such that its projection is equal to the reduced curve, i.e. $\Pi(\tilde{R}(t))=\Gamma(t)$. Then the curve $R(t)$ can be written as $R(t)=\Phi_{\theta(t)}(\tilde{R}(t))$ for some $\theta(t) \in S^{1}$. We find a differential equation for $\theta(t)$ so that the value of the momentum map for the reconstructed curve is conserved. In order to simplify the differential equation for $\theta(t)$, we restrict our discussion to the horizontal lift $R_{\text {hor }}(t)$, which is a particular reconstruction curve that has the additional property that $\mathcal{A}(\dot{\tilde{R}}(t))=0$, where $\mathcal{A}$ is the mechanical connection in (5.3).

Proposition 14 Suppose that the integral curve of the Lagrange-Routh reduced equation (5.7) is given by $(\Gamma(t), \dot{\Gamma}(t)) \in T S^{2}$ and the value of the momentum map $\mu$ is known. The following procedure reconstructs the motion of the $3 D$ pendulum to obtain $(R(t), \omega(t)) \in T S O(3)$ such that $\Pi(R(t))=\Gamma(t)$ and $\mathbf{J}(R(t), \omega(t))=\mu$.

1. Horizontally lift $\Gamma(t)$ to obtain $R_{\text {hor }}(t)$ by integrating the following equation with $R_{\text {hor }}(0)=R(0)$ :

$$
\dot{R}_{\text {hor }}(t)=R_{\text {hor }}(t) \hat{\omega}_{\text {hor }}(t),
$$

where

$$
\omega_{\mathrm{hor}}(t)=\dot{\Gamma}(t) \times \Gamma(t)-b(t) \Gamma(t) .
$$

2. Determine $\theta_{\mathrm{dyn}}(t) \in S^{1}$ from:

$$
\theta_{\mathrm{dyn}}(t)=\int_{0}^{t} \frac{\mu}{\Gamma(s) \cdot J \Gamma(s)} \mathrm{d} s .
$$


3. Reconstruct the desired curve in TSO(3) from:

$$
\begin{aligned}
& R(t)=\Phi_{\theta_{\mathrm{dyn}}(t)}\left(R_{\mathrm{hor}}(t)\right)=\exp \left[\theta_{\mathrm{dyn}}(t) \hat{e}_{3}\right] R_{\mathrm{hor}}(t), \\
& \omega(t)=\omega_{\mathrm{hor}}(t)+v(t) \Gamma(t)
\end{aligned}
$$

Proof See Appendix.

This leads to the geometric phase formula that expresses the rotation angle about the vertical axis along a closed integral curve of the Lagrange-Routh reduced equations.

Proposition 15 Assume the value of the momentum map $\mu=0$. Let $\Gamma(t)$, $t \in R$, define a closed curve in $S^{2}$, i.e. $\Gamma(0)=\Gamma(T)$ for some $T$. The geometric phase $\theta_{\mathrm{geo}}(T) \in S^{1}$ of the $3 D$ pendulum is defined by the relationship $R(T)=$ $\Phi_{\theta_{\text {geo }}(T)}(R(0))$ where

$$
\theta_{\text {geo }}(T)=\int_{\mathcal{B}} \frac{2\|J \Gamma(t)\|^{2}-\operatorname{tr}[J](\Gamma(t) \cdot J \Gamma(t))}{(\Gamma(t) \cdot J \Gamma(t))^{2}} \mathrm{~d} A,
$$

and $\mathcal{B}$ is a surface in $S^{2}$ with boundary $\Gamma(t)$.

\subsection{Integral of the Lagrange-Routh Reduced Model}

In this section we find an integral of motion for the Lagrange-Routh reduced model of the 3D pendulum, namely the total energy of the system. Note that the LagrangeRouth reduced equations of motion are derived by eliminating the conserved vertical component of the body-fixed angular momentum. In a later section, we make use of the constant energy surfaces to visualize the dynamics of the 3D pendulum.

Proposition 16 Assume the constant value of the momentum map is $\mu$. The total energy

$$
E=\frac{1}{2}(\dot{\Gamma} \times \Gamma+(v-b) \Gamma)^{T} J(\dot{\Gamma} \times \Gamma+(v-b) \Gamma)-m g \rho^{T} \Gamma
$$

is constant along solutions of the Lagrange-Routh reduced equations for the $3 D$ pendulum given by (5.7).

Proof Substituting the reconstruction equations for the angular velocity (5.11), (5.14) into the total energy expression (4.5), we obtain (5.16). The time derivative expression for the total energy is given by

$$
\dot{E}=(\dot{\Gamma} \times \Gamma+(v-b) \Gamma)^{T} J(\ddot{\Gamma} \times \Gamma+(\dot{v}-\dot{b}) \Gamma+(v-b) \dot{\Gamma})-m g \rho^{T} \dot{\Gamma} .
$$

Substituting the reduced equation of motion (5.7) into the above equation and rearranging, we can show that $\dot{E}=0$. 


\subsection{Equilibria of the Lagrange-Routh Reduced Model}

The Lagrange-Routh reduced model is related to the Lagrange-Poincaré reduced model through a projection of $T S O(3) / S^{1}$ onto $T S^{2}$. Thus, the equilibria structure of the Lagrange-Routh reduced model is equivalent to the Lagrange-Poincaré reduced model, but it is represented in terms of the reduced attitude $\Gamma_{\mathrm{e}}$ and the value of the momentum map $\mu$ instead of $\left(\Gamma_{\mathrm{e}}, \omega_{\mathrm{e}}\right)$.

We summarize the equilibria structure of the Lagrange-Routh reduced model using (5.7), showing its equivalence to the equilibria structure presented in Proposition 6.

Proposition 17 Consider the Lagrange-Routh reduced model of the $3 D$ pendulum given by (5.7). For the indicated values of the momentum map $\mu$, the Lagrange-Routh model on $T S^{2}$ has the following equilibria and relative equilibria:

1. If $\mu=0$ there is a hanging equilibrium: $\left(\frac{\rho}{\|\rho\|}, 0\right) \in T S^{2}$.

2. If $\mu=0$ there is an inverted equilibrium: $\left(-\frac{\rho}{\|\rho\|}, 0\right) \in T S^{2}$.

3. If the momentum map has one of the values

$$
\mu= \pm \sqrt{\frac{m g}{\left\|J^{-1} \rho\right\|^{3}}} \rho^{T} J^{-1} \rho,
$$

there is a relative equilibria in $T S^{2}$ :

$$
\left(-\frac{J^{-1} \rho}{\left\|J^{-1} \rho\right\|}, 0\right) \text {. }
$$

4. If the momentum map has one of the values

$$
\mu= \pm \sqrt{\frac{m g}{\left\|n_{\alpha}\right\|^{3}}} n_{\alpha}^{T} J n_{\alpha}
$$

there are one-dimensional relative equilibrium manifolds in $T S^{2}$ described by the parameterization:

$$
\left(-\frac{n_{\alpha}}{\left\|n_{\alpha}\right\|}, 0\right) \quad \text { for } \alpha \in \mathcal{L}_{i}, i \in\{1,2,3,4,5\},
$$

where $n_{\alpha}=\left(J-\frac{1}{\alpha} I_{3 \times 3}\right)^{-1} \rho \in \mathbb{R}^{3}$ and the intervals of the reals defined by

$$
\begin{aligned}
& \mathcal{L}_{1}=(-\infty, 0), \quad \mathcal{L}_{2}=\left(0, \frac{1}{J_{1}}\right), \quad \mathcal{L}_{3}=\left(\frac{1}{J_{1}}, \frac{1}{J_{2}}\right), \quad \mathcal{L}_{4}=\left(\frac{1}{J_{2}}, \frac{1}{J_{3}}\right), \\
& \mathcal{L}_{5}=\left(\frac{1}{J_{3}}, \infty\right) .
\end{aligned}
$$

Proof Substituting $\dot{\Gamma}_{\mathrm{e}}=0$ into (5.7)-(5.9), we obtain a condition for an equilibrium $\Gamma_{\mathrm{e}}$ :

$$
\Gamma_{\mathrm{e}} \times J^{-1}\left[v^{2} J \Gamma_{\mathrm{e}} \times \Gamma_{\mathrm{e}}-m g \Gamma_{\mathrm{e}} \times \rho\right]=0 .
$$


This is equivalent to

$$
\left[v^{2} J \Gamma_{\mathrm{e}} \times \Gamma_{\mathrm{e}}-m g \Gamma_{\mathrm{e}} \times \rho\right]=k_{2} J \Gamma_{\mathrm{e}}
$$

for some constant $k_{2} \in \mathbb{R}$. Taking the dot product of this and $\Gamma_{\mathrm{e}}$ implies that $0=$ $k_{2} \Gamma_{\mathrm{e}}^{T} J \Gamma_{\mathrm{e}}$. Since $\Gamma_{\mathrm{e}}^{T} J \Gamma_{\mathrm{e}}>0$ as the inertia tensor $J$ is positive definite and $\Gamma_{\mathrm{e}} \in S^{2}$, it follows that $k_{2}=0$. Thus, (5.21) is equivalent to

$$
v^{2} J \Gamma_{\mathrm{e}}+m g \rho=k_{1} \Gamma_{\mathrm{e}}
$$

for some constant $k_{1} \in \mathbb{R}$. Note that this is equivalent to the equilibrium condition for the Lagrange-Poincaré reduced model given by (4.9): for any solution $\left(\Gamma_{\mathrm{e}}, k, k_{1}\right)$ of (4.9), we can choose $\mu$ such that $k^{2}=v^{2}=\frac{\mu^{2}}{\left(\Gamma_{\mathrm{e}}^{T} J \Gamma_{\mathrm{e}}\right)^{2}}$, which gives a solution of (5.22), and vice versa. Thus, the equilibria structure of the Lagrange-Routh reduced model is equivalent to the equilibria of the Lagrange-Poincare reduced model. For an equilibrium $\left(\Gamma_{\mathrm{e}}, \omega_{\mathrm{e}}\right)$ of the Lagrange-Poincaré reduced model, the value of the momentum map at the corresponding equilibrium of the Lagrange-Routh model is given by

$$
\mu=k\left(\Gamma_{\mathrm{e}}^{T} J \Gamma_{\mathrm{e}}\right)=\omega_{\mathrm{e}}^{T} \Gamma_{\mathrm{e}}\left(\Gamma_{\mathrm{e}}^{T} J \Gamma_{\mathrm{e}}\right)
$$

Substituting this into the equilibria expressions in Proposition 6, we obtain the stated results.

According to Proposition 7, the following results hold for the Lagrange-Routh reduced model of the $3 \mathrm{D}$ pendulum.

Proposition 18 The equilibria of the $3 D$ pendulum given in the parameterizations (5.20) have the following convergence properties:

1. $\lim _{\alpha \rightarrow \infty} \Gamma_{e}=\Gamma_{\infty}$;

2. $\lim _{\alpha \rightarrow 0^{-}} \Gamma_{e}=\Gamma_{i}$

3. $\lim _{\alpha \rightarrow 0^{+}} \Gamma_{e}=\Gamma_{h}$;

4. for $i \in\{1,2,3\}: \lim \Gamma_{e}=\operatorname{sgn}\left(\rho_{i}\right) e_{i}$;

$\alpha \rightarrow \frac{1}{J_{i}}$

5. for $i \in\{1,2,3\}: \lim _{\alpha \rightarrow{\frac{1}{J_{i}}}^{+}} \Gamma_{e}=-\operatorname{sgn}\left(\rho_{i}\right) e_{i}$.

As previously, if additional assumptions are made about the location of the center of mass, then additional relative equilibria exist. The following results summarize this situation.

Proposition 19 Consider the 3D pendulum and assume that the location of the center of mass vector $\rho$ satisfies the indicated property. 
1. Assume there is exactly one index $i \in\{1,2,3\}$ for which $\rho_{i}=0$. If the momentum map has one of the values

$$
\mu= \pm \sqrt{\frac{m g}{\left\|p_{i}\right\|^{3}}} p_{i}^{T} J p_{i},
$$

there are one-dimensional relative equilibrium manifolds in $T S^{2}$ described by the parameterizations:

$$
\left(-\frac{p_{i}}{\left\|p_{i}\right\|}, 0\right) \quad \text { for any } \gamma \in \mathbb{R} \text {, }
$$

where $p_{1}=\left(\gamma, \frac{\rho_{2}}{J_{2}-J_{1}}, \frac{\rho_{3}}{J_{3}-J_{1}}\right), p_{2}=\left(\frac{\rho_{1}}{J_{1}-J_{2}}, \gamma, \frac{\rho_{3}}{J_{3}-J_{2}}\right), p_{3}=\left(\frac{\rho_{1}}{J_{1}-J_{3}}, \frac{\rho_{2}}{J_{2}-J_{3}}, \gamma\right)$.

2. Assume $\rho_{i}=0$ for exactly two indices $i \in\{1,2,3\}$. There are one-dimensional relative equilibrium manifolds in $T S^{2}$ described by the parameterizations:

$$
\left(e_{i}, 0\right), \quad\left(-e_{i}, 0\right) \quad \text { for any } \mu \in \mathbb{R} \text {. }
$$

This proposition includes the result for an asymmetric 3D pendulum that if the center of mass lies on a principal axis, then for any value of the angular momentum map there exist relative equilibria defined by a constant angular velocity vector that is aligned with that principal axis.

The geometric description of relative equilibria, based on the Lagrange-Routh model, provides additional insight into the equilibria structure of the $3 \mathrm{D}$ pendulum.

\subsection{Local Analysis of the Lagrange-Routh Reduced Model on $T S^{2}$}

We showed that when the angular momentum map is zero, $\mu=0$, the LagrangeRouth reduced model of the $3 \mathrm{D}$ pendulum has two isolated equilibria, namely the hanging equilibrium and the inverted equilibrium. These equilibria correspond to the disjoint equilibrium manifolds of the full equations of the $3 \mathrm{D}$ pendulum.

We next focus on these isolated equilibria of the Lagrange-Routh reduced equations. Using Proposition 17, the stability properties of the equilibrium manifolds of the $3 \mathrm{D}$ pendulum can be deduced by studying the Lagrange-Routh reduced equilibria for the case $\mu=0$. Compared to the Lagrange-Poincaré reduced model, the Lagrange-Routh reduction procedure results in a set of complicated equations that are a challenge to analyze.

Proposition 20 Assume the value of the momentum map is zero, $\mu=0$. The hanging equilibrium $\left(\Gamma_{h}, 0\right)=\left(\frac{\rho}{\|\rho\|}, 0\right)$ of the Lagrange-Routh reduced dynamics of the $3 D$ pendulum described by (5.7) is stable in the sense of Lyapunov.

Proof Consider the Lyapunov function

$$
V(\Gamma, \dot{\Gamma})=\frac{1}{2}(\dot{\Gamma} \times \Gamma+(v-b) \Gamma)^{\mathrm{T}} J(\dot{\Gamma} \times \Gamma+(v-b) \Gamma)+m g\left(\|\rho\|-\rho^{\mathrm{T}} \Gamma\right) .
$$


Note that $V\left(\Gamma_{h}, 0\right)=0$ and $V(\Gamma, \dot{\Gamma})>0$ elsewhere. Furthermore, the derivative along a solution of (4.3) and (4.4) is given by

$$
\dot{V}(\Gamma, \dot{\Gamma})=(\dot{\Gamma} \times \Gamma+(v-b) \Gamma)^{\mathrm{T}} J(\ddot{\Gamma} \times \Gamma+(\dot{v}-\dot{b}) \Gamma+(v-b) \dot{\Gamma})-m g \rho^{\mathrm{T}} \dot{\Gamma} .
$$

Substituting the reduced equation of motion (5.7) into the above equation and rearranging, we can show that $\dot{V}(\Gamma, \dot{\Gamma})=0$. Thus, the hanging equilibrium of (5.7) is Lyapunov stable.

Note that combining Proposition 20 with Proposition 17 immediately yields the result in Proposition 2.

The linearization of (5.7) at the inverted equilibrium can be shown to have two negative eigenvalues and two positive eigenvalues. Thus, the inverted equilibrium $\left(\Gamma_{i}, 0\right)$ of the Lagrange-Routh reduced model is unstable and locally there exists a two-dimensional stable manifold and a two-dimensional unstable manifold.

Proposition 21 Assume the value of the momentum map is zero, $\mu=0$. The inverted equilibrium $\left(\Gamma_{i}, 0\right)=\left(-\frac{\rho}{\|\rho\|}, 0\right)$ of the Lagrange-Routh reduced dynamics of the $3 D$ pendulum described by (5.7) is unstable.

Note that combining Proposition 21 with Proposition 17 immediately yields the result that the inverted equilibrium manifold I of the $3 \mathrm{D}$ pendulum given by (3.1)(3.2) is unstable.

\subsection{Poincaré Map on the Lagrange-Routh Reduced Model}

A Poincaré map describes the evolution of successive intersection points of a trajectory with a transversal hypersurface of codimension one. Typically, one chooses a hyperplane, and considers a trajectory with initial conditions on the hyperplane. The points at which this trajectory returns to the hyperplane are then observed, which provides insight into the stability of periodic orbits or the global characteristics of the dynamics.

The Lagrange-Routh reduced equations for the 3D pendulum on $T S^{2}$ are a particularly suitable choice for analysis using a Poincaré map, since it has dimension 4. On the manifold defined by a constant total energy as in (5.16) a Poincaré section on $T S^{2}$ defines a three-dimensional subspace of $T S^{2}$ on which the corresponding Poincaré map evolves. We define a Poincaré section on $T S^{2}$ for the Lagrange-Routh dynamics of the $3 \mathrm{D}$ pendulum given by (5.7) as follows.

$$
\mathcal{P}=\left\{(\Gamma, \dot{\Gamma}) \in T S^{2} \mid e_{3}^{T} \dot{\Gamma}=0, e_{3}^{T}(\Gamma \times \dot{\Gamma})>0, \text { and } E(\Gamma, \dot{\Gamma})=\mathrm{constant}\right\} .
$$

Suppose $\Gamma \in \mathcal{P}$ is given. The tangent space $T_{\Gamma} S^{2}$ is a plane that is tangential to $S^{2}$ and perpendicular to $\Gamma$. The first condition of the Poincaré section, $e_{3}^{T} \dot{\Gamma}=0$ determines a line in which the tangent vector $\dot{\Gamma} \in T_{\Gamma} S^{2}$ should lie, and the constraint of total energy conservation fixes the magnitude of the tangent vector in that line. Thus, the tangent vector is uniquely determined up to sign. The second condition of the Poincaré section resolves this ambiguity. It also excludes two reduced attitudes 
$\Gamma= \pm e_{3}$ for which the first condition is trivial; $e_{3}^{T} \dot{\Gamma}=0$ for any $\dot{\Gamma} \in T_{e_{3}} S^{2} \cup T_{-e_{3}} S^{2}$. Thus, $\mathcal{P}$ can be equivalently identified as

$$
\mathcal{P}=\left\{\Gamma \in S^{2} \mid e_{3}^{T} \dot{\Gamma}=0, e_{3}^{T}(\Gamma \times \dot{\Gamma})>0, \text { and } E(\Gamma, \dot{\Gamma})=\text { constant }\right\},
$$

where $(\Gamma, \dot{\Gamma})$ satisfies (5.7).

This Poincaré section in $T S^{2}$ is well defined in the sense that for each element, the corresponding tangent vector is uniquely determined. The attitude and the angular velocity in $T S O$ (3) can be obtained by using the reconstruction procedure for the given value of the momentum map $\mu$.

Although the Poincaré map is best explained in terms of the Lagrange-Routh reduced model as above, successive intersection points of a trajectory need not be computed directly from the complicated Lagrange-Routh reduced model. In particular, trajectories can be computed directly from the full 3D pendulum model given by (3.1) and (3.2) or from the Lagrange-Poincaré reduced model given by (4.3) and (4.4); the Poincaré map is obtained by a projection onto $S^{2}$.

\subsection{Visualization of Poincaré Map}

We examine the Poincaré map that describes dynamics features of a particular 3D pendulum model, demonstrating how this Poincaré map can be visualized. The $3 \mathrm{D}$ pendulum is chosen as an elliptic cylinder with properties of $m=1 \mathrm{~kg}, J=$ $\operatorname{diag}[0.13,0.28,0.17] \mathrm{kg} \cdot \mathrm{m}^{2}, \rho=[0,0,0.3] \mathrm{m}$. The initial condition are given by $R_{0}=I_{3 \times 3}$ and $\omega_{0}=c[1,1,1] \mathrm{rad} / \mathrm{s}$, where the constant $c$ is varied to give different total energy levels as specified in Fig. 2; the specific values of the momentum map are not required since the Lagrange-Poincaré equations need not be formed. The Lie group variational integrator introduced in Lee et al. (2005) is used to numerically integrate the full 3D pendulum equations (3.1) and (3.2), thereby obtaining the Poincaré map on $S^{2}$ numerically.

Since the center of mass lies on the third principal axis of the 3D pendulum, there is a relative equilibrium in which the angular velocity vector is aligned with the bodyfixed principal axis $e_{3}$ axis and the value of the angular momentum map is arbitrary. Figure 2 shows particular examples of the Poincaré map on $S^{2}$ corresponding to five different trajectories with various values of the total energy; the values of successive values of $\Gamma$ in $S^{2}$ are shown. Each of these trajectories can be viewed as a perturbation of a relative equilibrium whose angular velocity vector is along the principal axis $e_{3}$. In Fig. 2 the center of each Poincaré map is defined by the body-fixed principal axis $e_{3}$ representing this relative equilibrium. That is, the origin of the body-fixed axes in Fig. 2 is located at the center of $S^{2}$ with the $e_{1}$ axis pointing to the right in the plane of the page, the $e_{2}$ axis pointing to the top in the plane of the page, and $e_{3}$ is perpendicular to the plane of the page pointing outward.

It is interesting to see the transition of the Poincaré maps with varying total energy levels. The attitude dynamics of the 3D pendulum is periodic in Fig. 2(a), but it exhibits chaotic behavior with increased energy level in Figs. 2(b) and 2(c). If the total energy is increased further, the attitude dynamics becomes periodic again in Fig. 2(e). This demonstrates the highly nonlinear, and possibly chaotic, characteristics of the 3D pendulum dynamics. 

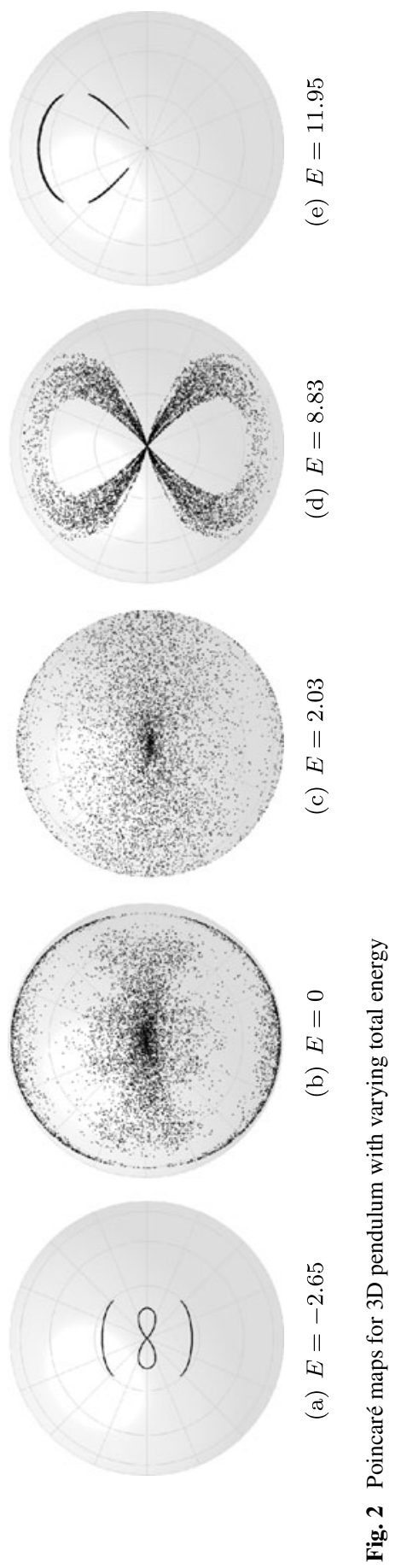


\section{Conclusions}

The asymmetric 3D pendulum, assuming the center of mass is distinct from the pivot location, exhibits rich dynamics with nontrivial geometric structure; this dynamics is much richer and more complex than the dynamics of a 1D planar pendulum, a 2D spherical pendulum, or any of the integrable cases such as the Lagrange top. This paper has demonstrated that the methods of geometric mechanics and the methods of nonlinear dynamics can be combined to obtain a unified perspective of the complex, non-integrable dynamics of the 3D pendulum.

The main contribution of the paper is that we have introduced three different models for the asymmetric 3D pendulum, including the full model defined on TSO(3), the Lagrange-Poincaré reduced model on $T S O(3) / S^{1}$ obtained by identifying configurations in the same group orbit, and the Lagrange-Routh reduced model on $T S^{2}$ where one additionally utilizes the fact that the dynamics evolves on a constant momentum level set. Relationships between the various representations are discussed in the context of conservation properties, equilibria and their stability properties, and invariant manifolds.

In addition, we illustrate that the use of the Lagrange-Routh reduced equations of motion, together with the energy conservation properties, allow the construction of a Poincaré map that can be readily visualized, thereby providing a graphical tool for obtaining insight into the rich nonlinear dynamical properties of the $3 \mathrm{D}$ pendulum.

Open Access This article is distributed under the terms of the Creative Commons Attribution Noncommercial License which permits any noncommercial use, distribution, and reproduction in any medium, provided the original author(s) and source are credited.

\section{Appendix}

In this appendix, we summarize Lagrange-Routh reduction and reconstruction procedures for the $3 \mathrm{D}$ pendulum.

\section{A.1 Reduction}

A description of Lagrange-Routh reduction can be found in Marsden et al. (2000) including expressions for the mechanical connection and the Routhian of the 3D pendulum given by (5.3) and (5.4), respectively. Here we derive the reduced equation of motion (5.7) using the Euler-Lagrange equation with magnetic terms for the given Routhian (5.4). These details are not provided in Marsden et al. (2000).

Variation of Routhian The Routhian satisfies the variational Euler-Lagrange equation with the magnetic term given by (5.6). We use a constrained variations of $\Gamma \in S^{2}$ :

$$
\begin{aligned}
& \delta \Gamma=\Gamma \times \eta, \\
& \delta \dot{\Gamma}=\dot{\Gamma} \times \eta+\Gamma \times \dot{\eta} .
\end{aligned}
$$


Here we assume that $\eta \cdot \Gamma=0$, since the component of $\eta$ parallel to $\Gamma$ has no effect on $\delta \Gamma$. These expressions are essential for developing the reduced equation of motion.

Using (A.1), (A.2), and the properties $\Gamma \cdot \dot{\Gamma}=0, \Gamma \cdot \eta=0$, the variation of the Routhian is given by

$$
\begin{aligned}
\delta R^{\mu}= & \dot{\eta} \cdot J(\dot{\Gamma} \times \Gamma-b \Gamma)-\eta \cdot \Gamma \times\left[-\dot{\Gamma} \times J(\dot{\Gamma} \times \Gamma)+\left(b^{2}+v^{2}\right) J \Gamma\right. \\
& -b J(\dot{\Gamma} \times \Gamma)+b(\dot{\Gamma} \times J \Gamma)+m g \rho] .
\end{aligned}
$$

Magnetic 2-form From the given mechanical connection $\mathcal{A}$ and a value of the momentum map $\mu \in \mathbb{R}^{*}$, define a 1 -form $\mathcal{A}_{\mu}$ on $T S O(3)$ by

$$
\mathcal{A}_{\mu}(R) \cdot(R, \hat{\omega})=\langle\mu, \mathcal{A}(R, \hat{\omega})\rangle=\mu \frac{e_{3}^{T} R J \omega}{e_{3}^{T} R J R^{T} e_{3}} .
$$

The magnetic 2-form $\beta_{\mu}$ in (5.5) is the exterior derivative of $\mathcal{A}_{\mu}$, which can be obtained by using the identity $\mathbf{d} \mathcal{A}_{\mu}(X, Y)=X\left[\mathcal{A}_{\mu}(Y)\right]-Y\left[\mathcal{A}_{\mu}(X)\right]-\mathcal{A}_{\mu}([X, Y])$ for $X=R \hat{\eta}, Y=R \hat{\zeta} \in T_{R} S O(3)$. Suppose that $\dot{\Gamma}=\Gamma \times \omega$. Since $\Gamma \cdot(\omega \times \eta)=$ $\eta \cdot(\Gamma \times \omega)=\eta \cdot \dot{\Gamma}$, the interior product of the magnetic 2 -form is given by

$$
\mathbf{i}_{\Gamma} \beta_{\mu}(\delta \Gamma)=\beta_{\mu}(\Gamma \times \omega, \Gamma \times \eta)=v\left\{\operatorname{tr}[J]-2 \frac{\|J \Gamma\|^{2}}{\Gamma \cdot J \Gamma}\right\} \dot{\Gamma} \cdot \eta,
$$

where $v=\frac{\mu}{\Gamma \cdot J \Gamma}$.

Euler-Lagrange Equation with Magnetic 2-form Substituting (A.3) and (A.4) into (5.6), and integrating by parts, the Euler-Lagrange equation for the reduced Routhian (5.4) is written as

$$
-\int_{0}^{T} \eta \cdot[J(\ddot{\Gamma} \times \Gamma-b \dot{\Gamma}-\dot{b} \Gamma)+\Gamma \times X+c \dot{\Gamma}] \mathrm{d} t=0
$$

where

$$
X=-\dot{\Gamma} \times J(\dot{\Gamma} \times \Gamma)+\left(b^{2}+v^{2}\right) J \Gamma-b J(\dot{\Gamma} \times \Gamma)+b(\dot{\Gamma} \times J \Gamma)+m g \rho,
$$

and $c$ is given by (5.9). Since (A.5) is satisfied for all $\eta$ with $\Gamma \cdot \eta=0$, we obtain

$$
J(\ddot{\Gamma} \times \Gamma-b \dot{\Gamma}-\dot{b} \Gamma)+\Gamma \times X+c \dot{\Gamma}=\lambda \Gamma,
$$

for $\lambda \in \mathbb{R}$. This is the reduced equation of motion. However, this equation has an ambiguity since the value of $\lambda$ is unknown; this equation is implicit for $\ddot{\Gamma}$ since the term $\dot{b}$ is expressed in terms of $\ddot{\Gamma}$. The next step is to determine expressions for $\lambda$ and $\dot{b}$ using the definition of $b$ and some vector identities.

We first find an expression for $\lambda$ in terms of $\Gamma, \dot{\Gamma}$. Taking the dot product of (A.7) with $\Gamma$, we obtain

$$
\Gamma \cdot J(\ddot{\Gamma} \times \Gamma-b \dot{\Gamma}-\dot{b} \Gamma)=\lambda
$$


From the definition of $b$, we obtain the following identity: $\Gamma \cdot J(\dot{\Gamma} \times \Gamma-b \Gamma)=0$. Differentiating this with respect to time and substituting into (A.8), we find an expression for $\lambda$ in terms of $\Gamma, \dot{\Gamma}$ as

$$
\lambda=-\dot{\Gamma} \cdot J(\dot{\Gamma} \times \Gamma-b \Gamma) .
$$

Substituting (A.9) into (A.7), and taking the dot product of the result with $\Gamma$, we obtain an expression for $\dot{b}$ in terms of $\Gamma, \dot{\Gamma}$ as

$$
\dot{b}=\Gamma \cdot J^{-1}\{\Gamma \times X+c \dot{\Gamma}+(\dot{\Gamma} \cdot J(\dot{\Gamma} \times \Gamma-b \Gamma)) \Gamma\} .
$$

Substituting (A.10) into (A.7), and using the vector identity $Y-(\Gamma \cdot Y) \Gamma=(\Gamma$. $\Gamma) Y-(\Gamma \cdot Y) \Gamma=-\Gamma \times(\Gamma \times Y)$ for any $Y \in \mathbb{R}^{3}$, we obtain the following form for the reduced equation of motion:

$$
\ddot{\Gamma} \times \Gamma-b \dot{\Gamma}-\Gamma \times\left[\Gamma \times J^{-1}\{\Gamma \times X+c \dot{\Gamma}+(\dot{\Gamma} \cdot J(\dot{\Gamma} \times \Gamma-b \Gamma)) \Gamma\}\right]=0 .
$$

Reduced Equation of Motion This equation has no ambiguity. Now, we simplify this equation. The above expression is equivalent to the following equation

$$
\begin{aligned}
& \Gamma \times\left[\ddot{\Gamma} \times \Gamma-b \dot{\Gamma}-\Gamma \times\left[\Gamma \times J^{-1}\{\Gamma \times X+c \dot{\Gamma}+(\dot{\Gamma} \cdot J(\dot{\Gamma} \times \Gamma-b \Gamma)) \Gamma\}\right]\right] \\
& \quad=0 .
\end{aligned}
$$

Since $\Gamma \cdot \ddot{\Gamma}=-\|\dot{\Gamma}\|^{2}$, the first term is given by

$$
\Gamma \times(\ddot{\Gamma} \times \Gamma)=(\Gamma \cdot \Gamma) \ddot{\Gamma}-(\Gamma \cdot \ddot{\Gamma}) \Gamma=\ddot{\Gamma}+\|\dot{\Gamma}\|^{2} \Gamma .
$$

Using the property $\Gamma \times(\Gamma \times(\Gamma \times Y))=-(\Gamma \cdot \Gamma) \Gamma \times Y=-\Gamma \times Y$ for $Y \in \mathbb{R}^{3}$, the third term of the above equation can be simplified. Substituting (A.6) and rearranging, the reduced equation of motion for the $3 \mathrm{D}$ pendulum is given by

$$
\ddot{\Gamma}=-\|\dot{\Gamma}\|^{2} \Gamma+\Gamma \times \Sigma,
$$

where $\Sigma=b \dot{\Gamma}+J^{-1}\left[(J(\dot{\Gamma} \times \Gamma)-b J \Gamma) \times((\dot{\Gamma} \times \Gamma)-b \Gamma)+v^{2} J \Gamma \times \Gamma-m g \Gamma \times\right.$ $\rho-c \dot{\Gamma}]$.

\section{A.2 Reconstruction}

For a given integral curve of the reduced equation $(\Gamma(t), \dot{\Gamma}(t)) \in T S^{2}$, we find a curve $\tilde{R}(t) \in S O(3)$ that is projected into the reduced curve, i.e. $\Pi(\tilde{R}(t)=\Gamma(t)$. The reconstructed curve can be written as $R(t)=\Phi_{\theta(t)}(\tilde{R}(t))$ for some $\theta(t) \in S^{1}$. The conservation of the momentum map yields the following reconstruction equation (Marsden et al. 2000)

$$
\theta(t)^{-1} \dot{\theta}(t)=\mathbb{I}^{-1}(\tilde{R}(t)) \mu-\mathcal{A}(\dot{\tilde{R}}(t))
$$

The particular choice of $\tilde{R}(t)$, the horizontal lift given by (5.10), simplifies the above equation, since the horizontal part of the tangent vector is annihilated by the mechanical connection, and as such the second term in the above equation vanishes. 
Furthermore, since the group $S^{1}$ is abelian, the solution reduces to a quadrature as in (5.12). The reconstructed curve is given by (5.13).

\section{References}

Arnold, V.I., Kozlov, V.V., Neishtadt, A.I.: Dynamical Systems III. Springer, Berlin (1988)

Astrom, K.J., Furuta, K.: Swinging up a pendulum by energy control. Automatica 36, 287-295 (2000)

Bernstein, D.S., McClamroch, N.H., Bloch, A.M.: Development of air spindle and triaxial air bearing testbeds for spacecraft dynamics and control experiments. In: Proceedings of the American Control Conference, pp. 3967-3972 (2001)

Bloch, A.M.: Nonholonomic Mechanics and Control. Springer, Berlin (2003)

Bruno, A.D.: Analysis of the Euler-Poisson equations by methods of power geometry and normal form. J. Appl. Math. Mech. 71, 168-199 (2007)

Cendra, H., Marsden, J.E., Ratiu, T.S.: Lagrangian reduction by stages. Mem. Am. Math. Soc. 152(722), $1-108(2001)$

Chaturvedi, N.A., McClamroch, N.H.: Asymptotic stabilization of the hanging equilibrium manifold of the 3D pendulum. Int. J. Robust Nonlinear Control 17, 1435-1454 (2007)

Cho, S., Shen, J., McClamroch, N.H., Bernstein, D.S.: Equations of motion of the triaxial attitude control testbed. In: Proceedings of the IEEE Conference on Decision and Control, pp. 3429-3434 (2001)

Cho, S., Shen, J., McClamroch, N.H.: Mathematical models for the triaxial attitude control testbed. Math. Comput. Model. Dyn. Syst. 9, 165-192 (2003)

Cushman, R.H., Bates, L.M.: Global Aspects of Classical Integrable Systems. Birkhäuser, Basel (1997)

Furuta, K.: Control of pendulum: from super mechano-system to human adaptive mechatronics. In: Proceedings of the IEEE Conference on Decision and Control, pp. 1498-1507 (2003)

Hernández-Garduño, A., Lawson, J.K., Marsden, J.E.: Relative equilibria for the generalized rigid body. J. Geom. Phys. 53, 259-274 (2005)

Holmes, P.J., Marsden, J.E.: Horseshoes and Arnold diffusion for Hamiltonian systems on Lie groups. Indiana Univ. Math. J. 32, 962-967 (1983)

Karapetyan, A.V.: Invariant sets in the Goryachev-Chaplygin problem: existence, stability, and branching. J. Appl. Math. Mech. 70, 195-198 (2006)

Lee, T., Leok, M., McClamroch, N.H.: A Lie group variational integrator for the attitude dynamics of a rigid body with application to the 3D pendulum. In: Proceedings of the IEEE Conference on Decision and Control, pp. 962-967 (2005)

Lewis, D., Ratiu, T.S., Simo, J.C., Marsden, J.E.: The heavy top: a geometric treatment. Nonlinearity 5, $1-48(1992)$

Maddocks, J.H.: Stability of relative equilibria. IMA J. Appl. Math. 46, 71-99 (1991)

Marsden, J.E., Ratiu, T.S., Scheurle, J.: Reduction theory and the Lagrange-Routh equations. J. Math. Phys. 41, 3379-3429 (2000)

Shen, J., Sanyal, A.K., Chaturvedi, N.A., Bernstein, D.S., McClamroch, N.H.: Dynamics and control of a 3D pendulum. In: Proceedings of the IEEE Conference on Decision and Control, pp. 323-328 (2004) 